\title{
CRITERIA FOR VERY AMPLENESS OF RANK TWO VECTOR BUNDLES OVER RULED SURFACES
}

\author{
ALBERTO ALZATI AND GIAN MARIO BESANA
}

\begin{abstract}
Very ampleness criteria for rank 2 vector bundles over smooth, ruled surfaces over rational and elliptic curves are given. The criteria are then used to settle open existence questions for some special threefolds of low degree.
\end{abstract}

\section{INTRODUCTION.}

A vector bundle $\mathcal{E}$ over a smooth algebraic variety $Y$ is said to be very ample if the tautological line bundle $\mathcal{O}_{\mathbb{P}(\mathcal{E})}(1)$ is very ample on the projectivized bundle $\mathbb{P}(\mathcal{E})$. Very ampleness of $\mathcal{E}$ is therefore equivalent to the existence of a projective smooth manifold $X=\mathbb{P}(\mathcal{E})$ embedded as a linear scroll on $Y$.

Although it is in general impossible to give a numerical characterization of very ampleness, one can try to find sufficient numerical conditions to guarantee it when the Picard group of $Y$ is particularly simple.

In this paper some classical ideas are revisited in order to give some very ampleness criteria for rank 2 vector bundles $\mathcal{E}$ over smooth, ruled surfaces on rational and elliptic curves.

In Section 3, classical ideas on obtaining very ampleness criteria by lifting of sections from appropriately chosen divisors are revisited in our context. They are applied to obtain the very ampleness of a family of rank 2 vector bundles over $\mathbb{P}^{2}$, see Corollary 3.2 , and, in particular, an existence result for 3-dimensional scrolls over $\mathbb{P}^{2}$, of degree 11 and genus 6 , left as an open question in [B-B-1], see Remark 4. Section 4 presents a purely numerical very ampleness criterion for rank 2 vector bundles over rational ruled surfaces, see Theorem 4.2, with an example of its application. Section 5 deals with the case of ruled surfaces over elliptic curves. Section 6 contains a very ampleness criterion criterion for a very special class of vector bundles $\mathcal{E}$ on $\mathbf{F}_{1}$, the Hizebruch surface with invariant $e=1$. The criterion is partially based upon a new observation that relates the very ampleness of $\mathcal{E}$ with whether points in the zero locus of a generic section of $\mathcal{E}$ are in general position on $Y$. Results from section 6 are applied to establish further existence and non existence results for threefolds scrolls over $\mathbf{F}_{1}$, of low degree, previously left as open problems in [F-L-2] and [B-B-1].

Date: february, 122009.

1991 Mathematics Subject Classification. Primary 14E05, 14J30.

Key words and phrases. vector bundles, very ampleness, ruled surfaces.

This work is within the framework of the national research project "Geomety of Algebraic Varieties" Cofin 2006 of MIUR. 
Acknowledgements: The authors wish to thank E. Arrondo for some helpful conversations around the zero locus of generic sections of rank 2 vector bundles over surfaces and the referee for the very careful revision of the paper.

\section{Notation AND BACKGround MATERIAL}

The ground field is fixed to be $\mathbb{C}$, and $\mathbb{P}^{n}$ denotes the $n$-dimensional complex projective space. The focus of this work is on rank-two vector bundles over smooth surfaces. In this context we fix the following notation:

- $\mathcal{E}$ : a rank- $r$ vector bundle over a smooth variety $Y, \operatorname{dim} Y \geq 2$;

- $c_{i}(\mathcal{E})$ : the $i$-th Chern class of $\mathcal{E}$;

- $X=\mathbb{P}(\mathcal{E})$ : the projectivization of $\mathcal{E}$;

- $\pi: \mathbb{P}(\mathcal{E}) \rightarrow Y$ the natural projection onto the base;

- $T$ : the tautological line bundle of $X$, i.e. $\mathcal{O}_{X}(T)=\mathcal{O}_{\mathbb{P}(\mathcal{E})}(1)$;

- $|T|$ : linear system of effective divisors linearly equivalent to $T$;

- $\Gamma$ : projectivization of the restriction $\mathcal{E}_{\mid \gamma}$, where $\gamma$ is a smooth curve on $Y$;

- $\mathbf{F}_{e}$ : rational ruled surface of invariant $e \geq 0$, i.e. $\mathbb{P}\left(\mathcal{O}_{\mathbb{P}^{1}} \oplus \mathcal{O}_{\mathbb{P}^{1}}(-e)\right)$;

- $\rho: \mathbf{F}_{e} \rightarrow \mathbb{P}^{1}$ : the natural projection onto the base;

- $C_{0}, f$ : standard generators of $\operatorname{Num}\left(\mathbf{F}_{e}\right) \simeq \operatorname{Pic}\left(\mathbf{F}_{e}\right)$;

- $F$ : projectivization of the restriction $\mathcal{E}_{\mid f}$ where $f$ is a fibre of $Y$ when $Y=\mathbf{F}_{e}$

- $(\sigma)_{0}$ : zero locus of the section $\sigma$ of a vector bundle;

- $\equiv$ : numerical equivalence of divisors;

- $K_{S}$ : canonical divisor of the smooth surface $S$;

- $X^{[t]}$ : the Hilbert scheme of zero-subschemes of $X$ of length $t$.

- $\mathbb{T}_{P}(M)$ : the holomorphic tangent space to an analytic manifold $M$ at a point $P$.

Cartier divisors on smooth projective varieties, their associated line bundles and invertible sheaves of their holomorphic sections are used with no distinction. Mostly additive notation is used for their group. Given a divisor (line bundle) $D$ on a smooth projective variety, $|D|$ denotes the complete linear system of effective divisors linearly equivalent (associated) to $D$. Given any subvariety $S$ in $X$ and a line bundle $L \in \operatorname{Pic}(X)$, we denote by $L_{\left.\right|_{S}}$ the restriction of $L$ to $S$, i.e. $L_{\left.\right|_{S}}=L \otimes \mathcal{O}_{S}$.

Let $X$ and $T$ be as above. For any smooth surface $\Sigma$ contained in $X$ let $\left|T_{\mid \Sigma}\right|$ be the complete linear system associated to $T_{\mid \Sigma}$, i.e. given by $H^{0}\left(T \otimes \mathcal{O}_{\Sigma}\right)$. Let $|T|_{\mid \Sigma}$ be the restriction of the linear system $|T|$ to $\Sigma$, i.e. given by the image of the restriction map $r: H^{0}(X, T) \rightarrow H^{0}\left(\Sigma, T_{\left.\right|_{\Sigma}}\right)$. Then $\left|T_{\mid \Sigma}\right| \supseteq|T|_{\mid \Sigma}$ and equality holds if $h^{1}(X, T-\Sigma)=0$.

If $\Sigma$ is reducible as the union of two smooth surfaces $S_{1} \cup S_{2}$, intersecting transversely only along a smooth (possibly reducible) curve $C$, it is: $\left\{\left(\sigma_{1}, \sigma_{2}\right)\left|\sigma_{i} \in\right| T_{\mid S_{i}} \mid\right.$ $\left.i=1,2, \sigma_{1_{\mid C}}=\sigma_{2_{\mid C}}\right\}=\left|T_{\mid \Sigma}\right| \subseteq\left|T_{\mid S_{1}}\right| \oplus\left|T_{\mid S_{2}}\right|=\left\{\left(\sigma_{1}, \sigma_{2}\right)\left|\sigma_{i} \in\right| T_{\mid S_{i}} \mid i=1,2\right\}$, while $|T|_{\mid \Sigma}=\left\{\left(\sigma_{1}, \sigma_{2}\right)\left|\sigma_{i} \in\right| T_{\mid S_{i}} \mid i=1,2\right.$ and there exists $\tau \in|T|$ such that $\tau_{\mid S_{i}}=\sigma_{i}$ $i=1,2\}$.

Let $\xi \in X^{[t]}$. A subvariety $S$ is said to pass through $\xi$ if and only if $S$ contains $\xi$ scheme theoretically. If $t=\operatorname{length}(\xi)=2$ and $\operatorname{Supp}(\xi)$ consists of two distinct points, scheme theoretic inclusion is equivalent to ordinary inclusion. If $t=\operatorname{length}(\xi)=2, \operatorname{Supp}(\xi)$ consists of one point $P$, and $X$ and $S$ are smooth at 
$P$, let $\underline{q} \in \mathbb{T}_{P}(X)$ denote the tangent direction at $P$ specified by $\xi$. Then scheme theoretic inclusion is equivalent to $P \in S$ and $q \in \mathbb{T}_{P}(S)$.

Let $L$ be a line bundle on a variety $X$ and let $\xi \in X^{[t]}$. Let $V \subset H^{0}(L)$ be a subspace of sections and let $|V|$ be the associated linear system. The expression $|V|$ separates $\xi$ is used to mean that the restriction map $V \rightarrow H^{0}(\xi)$ is surjective. In this language a line bundle $L$ is very ample if and only if the associated complete linear system separates every $\xi \in X^{[2]}$.

If $\xi \in X^{[t]}$ is a reduced, 0 -dimensional, scheme, we often identify the scheme itself with its support. For example we refer to "points of $\xi$ " to mean points of $\operatorname{Supp}(\xi)$.

Lemma 2.1. Let $Y$ be a ruled surface of invariant e, over a smooth curve $C$ of genus $g$. Let $x$ be an integer and assume the line bundle $C_{0}+x f$ is very ample. If $\ell \subset Y$ is a line on $Y$ in the embedding given by $\left|C_{0}+x f\right|$, then either $\ell=f$ or $g=0, x=e+1$, and $\ell=C_{0}$.

Proof. As $C_{0}+x f$ is very ample, it is in particular ample and therefore, (see [H, Corollary V.2.18, Proposition V.2.20, Proposition V.2.21]) it must be

$$
x>\left\{\begin{array}{l}
e \text { if } e \geq 0 \\
\frac{e}{2} \text { if } e<0 .
\end{array}\right.
$$

For an irreducible divisor $\ell \in\left|a C_{0}+b f\right|$ to be a line in the embedding given by $C_{0}+x f$ it must be $\left(C_{0}+x f\right)\left(a C_{0}+b f\right)=1$ i.e.

$$
1=a x-a e+b .
$$

By considering that the arithmetic genus of $\ell$ must be zero, one can easily check that the necessary conditions for $\ell$ to be an irreducible divisor, contained in $[\mathrm{H}$, Corollary 2.18, Proposition 2.20, Proposition 2.21], are incompatible with (1), (2), and the very ampleness of $C_{0}+x f$ unless $\ell$ is as in the statement.

\section{Classical ideas}

The following notation will be fixed throughout the paper. Let $Y$ be a smooth algebraic surface. Let $\mathcal{E}$ be a rank 2 vector bundle over $Y$, and let $X=\mathbb{P}(\mathcal{E})$. Let $\pi: X \rightarrow Y$ be the natural projection and let $T$ be the tautological line bundle. Let $A \in \operatorname{Pic}(Y)$ and let $D=T+\pi^{*} A \in \operatorname{Pic}(X)$. The first Proposition in this section is a simple adaptation to our context of a classical lifting of sections approach to prove very ampleness.

Proposition 3.1. With the notation fixed in this section, let $D_{\epsilon}=\epsilon T+\pi^{*}(A)$, where $\epsilon=0,1$ and assume:

a) for all $\xi \in X^{[2]}$ there exists a smooth, irreducible element $S \in\left|D_{\epsilon}\right|$, passing through $\xi$

b) $h^{1}\left(X,(1-\epsilon) T-\pi^{*}(A)\right)=0$;

c) $T_{\mid S}$ is very ample on $S$.

Then $T$ is very ample on $X$.

Proof. Given any $\xi \in X^{[2]}$, assumption a) gives an element $S \in\left|D_{\epsilon}\right|$ passing through it. By assumption c) we can separate $\xi$ on $S$ by elements of $\left|T_{\mid S}\right|$. Consider the sequence: $0 \rightarrow H^{0}\left(X, T-D_{\epsilon}\right) \rightarrow H^{0}(X, T) \rightarrow H^{0}\left(S, T_{\mid S}\right) \rightarrow H^{1}\left(X, T-D_{\epsilon}\right)=$ $H^{1}\left(X,(1-\epsilon) T-\pi^{*} A\right)=0$. Assumption b) allows us to lift the separating sections 
on $S$ to sections of $T$ separating $\xi$ on $X$. Notice that when $\epsilon=1$ assumption b) can be restated as $h^{1}(Y,-A)=0$.

As an immediate application of Proposition 3.1, when $\epsilon=0$, one obtains the very ampleness of a family of rank- 2 vector bundles over $\mathbb{P}^{2}$.

Corollary 3.2. There exists very ample vector bundles $\mathcal{E}$ of rank 2 over $Y=\mathbb{P}^{2}$ given by non trivial extensions

$$
0 \rightarrow \mathcal{O}_{\mathbb{P}^{2}}(1) \rightarrow \mathcal{E} \rightarrow \mathcal{O}_{\mathbb{P}^{2}}(4) \otimes \mathcal{I}_{\eta} \rightarrow 0,
$$

where $\eta \in \mathbb{P}^{2[10]}$ consists of 10 distinct points in general position.

Proof. Let $\eta \in \mathbb{P}^{2}{ }^{[10]}$ consist of 10 distinct points in general position. Notice that $\operatorname{Ext}^{1}\left(\mathcal{O}_{\mathbb{P}^{2}}(3) \otimes \mathcal{I}_{\eta}, \mathcal{O}_{\mathbb{P}^{2}}\right) \neq 0$ and there exists a locally free extension

$$
0 \rightarrow \mathcal{O}_{\mathbb{P}^{2}} \rightarrow \mathcal{E}^{\prime} \rightarrow \mathcal{O}_{\mathbb{P}^{2}}(3) \otimes \mathcal{I}_{\eta} \rightarrow 0,
$$

because $K_{Y} \otimes \mathcal{O}_{\mathbb{P}^{2}}(3)=\mathcal{O}_{\mathbb{P}^{2}}$, so that, for any point $w \in \eta$, the natural map $0=H^{0}\left(Y, \mathcal{I}_{\eta}\right)=H^{0}\left(Y, K_{Y} \otimes \mathcal{O}_{\mathbb{P}^{2}}(3) \otimes \mathcal{I}_{\eta}\right) \rightarrow H^{0}\left(Y, K_{Y} \otimes \mathcal{O}_{\mathbb{P}^{2}}(3) \otimes \mathcal{I}_{\eta \backslash w}\right)=$ $H^{0}\left(Y, \mathcal{I}_{\eta \backslash w}\right)=0$ is an isomorphism as required, see [D-L], Theorem 3.13 and its proof. Twisting $(3)$ by $\mathcal{O}_{\mathbb{P}^{2}}(1)$ and setting $\mathcal{E}=\mathcal{E}^{\prime}(1)$ gives

$$
0 \rightarrow \mathcal{O}_{\mathbb{P}^{2}}(1) \rightarrow \mathcal{E} \rightarrow \mathcal{O}_{\mathbb{P}^{2}}(4) \otimes \mathcal{I}_{\eta} \rightarrow 0 .
$$

Notice that $c_{1}(\mathcal{E})=\mathcal{O}_{\mathbb{P}^{2}}(5)$, and $c_{2}(\mathcal{E})=14$. Let $D=\pi^{*}\left(\mathcal{O}_{\mathbb{P}^{2}}(1)\right)$, i.e. $\epsilon=0$ and $A=\mathcal{O}_{\mathbb{P}^{2}}(1)$ in the notation of Proposition 3.1. Let $\mathcal{E}_{\left.\right|_{\ell}}$ be the restriction of $\mathcal{E}$ to any line $\ell$. From (4) one gets:

$$
0 \rightarrow \mathcal{O}_{\ell}(1+\varepsilon) \rightarrow \mathcal{E}_{\mid \ell} \rightarrow \mathcal{O}_{\ell}(4-\varepsilon) \rightarrow 0,
$$

where $\varepsilon=0,1,2$, respectively, if $\ell$ passes through $0,1,2$ points of $\eta$. Sequence (5) shows that $\mathcal{E}_{\left.\right|_{\ell}}$ is very ample, so that assumptions a) and c) of Proposition 3.1 are satisfied. To verify assumption b) of Proposition 3.1 notice that $h^{1}(X, T-D)=$ $h^{1}\left(\mathbb{P}^{2}, \mathcal{E}(-1)\right)=h^{1}\left(\mathbb{P}^{2}, \mathcal{E}^{\prime}\right)$, which, looking at sequence $(3)$, vanishes if and only if $h^{1}\left(\mathbb{P}^{2}, \mathcal{O}_{\mathbb{P}^{2}}(3) \otimes \mathcal{I}_{\eta}\right)$ vanishes. As $\eta$ consists of 10 points in general position it is $H^{0}\left(\mathcal{O}_{\mathbb{P}^{2}}(3) \otimes \mathcal{I}_{\eta}\right)=0$ and thus the sequence

$$
0 \rightarrow \mathcal{O}_{\mathbb{P}^{2}}(3) \otimes \mathcal{I}_{\eta} \rightarrow \mathcal{O}_{\mathbb{P}^{2}}(3) \rightarrow \mathcal{O}_{\mathbb{P}^{2}}(3) \otimes \mathcal{O}_{\eta} \rightarrow 0
$$

gives the required vanishing.

Remark 3.1. Corollary 3.2 implies the existence of a family of 3-dimensional scrolls $(X, L)=\left(\mathbb{P}(\mathcal{E}), \mathcal{O}_{\mathbb{P}(\mathcal{E})}(1)\right)$ embedded by $\left|\mathcal{O}_{\mathbb{P}(\mathcal{E})}(1)\right|$ in $\mathbb{P}^{7}$ with $\operatorname{deg}(X)=$ $\left[c_{1}(\mathcal{E})\right]^{2}-c_{2}(\mathcal{E})=11$, and sectional genus $g(X)=6$ (see also Section 7$)$. The existence of such threefolds was left as an open question in [B-B-1], Proposition 4.2.3. The Hilbert scheme of a threefold $X \subset \mathbb{P}^{7}$ as in Corollary 3.2 has an irreducible component of dimension 83, of which $X$ is a smooth point. See [B-F], Proposition 3.1, Corollary 3.3 and Remark 3.4, for details.

Without any assumption on the positivity of $D$ it would be very difficult to establish whether assumption a) in Proposition 3.1 is satisfied or not. If $D$ were very ample it is well known, [B-S], that if there is a zero scheme $\xi$ for which a) fails, then the ambient variety is a surface containing a $D$-line $\ell$ through $\xi$, such that all divisors in $|D|$ passing through $\xi$ are reducible as $\ell+D^{\prime}$. In our particular situation, keeping in mind that we are striving for sufficient numerical criteria, we 
can relax conditions on $D$ as in the following Proposition. Before proving it we need a Lemma.

Lemma 3.3. With the notation fixed in this Section, let $\mathrm{F}$ be the class of a generic fibre of $\pi$ in the Chow ring of $X$. Let $\Sigma$ be a singular element in $|T|$. Then $\Sigma$ is reducible, more precisely $\Sigma=\pi^{-1}(Z) \cup \Sigma^{\prime}$ where $Z$ is an effective divisor on $Y$ and $\Sigma^{\prime} \cdot \mathrm{F}=1$, and if $Z$ is maximal with respect to the previous decomposition then $\Sigma^{\prime}$ is smooth and irreducible.

Proof. Let $R$ be a singular point of $\Sigma$ and let $\mathrm{F}_{R}$ be the fibre of $\pi$ passing through $R$. Then $\mathrm{F}_{R} \simeq \mathbb{P}^{1}$ should intersect $\Sigma$ in $R$ with multiplicity at least 2 . As $\Sigma \cdot \mathrm{F}_{R}=1$ in the Chow ring of $X$, this is possible only if $\mathrm{F}_{R}$ is contained in $\Sigma$. If $\mathrm{F}_{R}$ is an isolated fibre contained in $\Sigma$ then $\Sigma$ would be, locally, the blow up of $Y$ at $\pi(R)$, hence it would be smooth at $R$. It follows that there are infinitely many fibres of $\pi$ contained in $\Sigma$. So that we can write $\Sigma=\pi^{-1}(Z) \cup \Sigma^{\prime}$ where $Z$ is a suitable effective divisor on $Y$. Obviously $1=\Sigma \cdot \mathrm{F}=\Sigma^{\prime} \cdot \mathrm{F}$. If $\Sigma^{\prime}$ is smooth and irreducible we are done, otherwise we can argue as before for $\Sigma^{\prime}$ until we get a decomposition with a smooth $\Sigma^{\prime} \in\left|T-\pi^{*} Z\right|$. Such a $\Sigma^{\prime}$ is also irreducible thank to the maximality of $Z$.

Proposition 3.4. With the notation fixed in this Section, assume:

a) $h^{0}(X, D)>3$

b) for all $B \in \operatorname{Pic}(Y)$ such that $T+\pi^{*} B$ is effective and $A-B$ is effective, then $\max \left\{h^{0}\left(X, T+\pi^{*} B\right), h^{0}(Y, A-B)\right\}<h^{0}(X, D)-2$.

Then assumption a) of Proposition 3.1 is satisfied with $\varepsilon=1$.

Proof. As $h^{0}(X, D)>3$, for all $\xi \in X^{[2]}$ the linear system $|V|=\left|D \otimes \mathcal{I}_{\xi}\right|$ is not empty. Let $S$ be an element of $|V|$. If $S$ is smooth we have nothing to prove, otherwise, by applying Lemma 3.3 to $\mathcal{E} \otimes \mathcal{O}_{Y}(A)$, we can write $S=\pi^{-1}\left(A^{\prime}\right) \cup S^{\prime}$ where $S^{\prime} \in\left|T+\pi^{*} B\right|$ is effective, irreducible and smooth for some $B \in \operatorname{Pic}(Y)$ and where $A^{\prime} \in|A-B|$ is effective on $Y$.

Notice that $\operatorname{dim}(|V|) \geq \operatorname{dim}(|D|)-2$. It is enough to show that not every element of $|V|$ is singular. By contradiction, let us assume that all elements $S$ of $|V|$ are singular. By Bertini's Theorem $|V|$ has a base locus E and for any generic $S \in|V|$ we have that $\operatorname{Sing}(S) \subseteq$ E. On the other hand we know that $S=\pi^{-1}\left(A^{\prime}\right) \cup S^{\prime}$ as above and $S$ is singular along $\pi^{-1}\left(A^{\prime}\right) \cap S^{\prime}$ which can not be the union of a finite number of fibres and can not contain isolated fibres (see the proof of Lemma 3.3), so that there is a maximal curve $C_{S} \subseteq \operatorname{Sing}(S) \subseteq \mathrm{E}$ such that $\pi[\operatorname{Sing}(S)]=\pi\left(C_{S}\right)$, $\operatorname{dim} \pi\left(C_{S}\right)=1, \pi\left(C_{S}\right) \subseteq A^{\prime}$ and $\pi^{-1}\left[\pi\left(C_{S}\right)\right]=\pi^{-1}\left(A^{\prime}\right)$; hence $\operatorname{dim}(\mathrm{E}) \geq 1$ and $\operatorname{dim}[\pi(\mathrm{E})] \geq 1$. Let us consider $\pi(\mathrm{E})$.

If $\operatorname{dim}[\pi(\mathrm{E})]=2$ then $\operatorname{dim}(\mathrm{E})=2$ and $|V|=\left(T+\pi^{*} B\right)+\left|\pi^{*} A-\pi^{*} B\right|$, where $B$ is a suitable divisor of $Y$ such that $T+\pi^{*} B$ is effective on $X$, where $A-B$ is effective on $Y$ and where $\left(T+\pi^{*} B\right)$ is in the fixed part of $|V|$. But this is not possible, by assumption b), as $\operatorname{dim}\left(\left|\pi^{*} A-\pi^{*} B\right|\right)=\operatorname{dim}(|V|) \geq \operatorname{dim}(|D|)-2$.

If $\operatorname{dim}[\pi(\mathrm{E})]=1$ let us consider the above curves $C_{S} \subseteq \mathrm{E}$ for any generic $S \in|V|$. If $C_{S}=C_{\bar{S}}$ for some fixed $\bar{S}=\pi^{-1}\left(\bar{A}^{\prime}\right) \cup \bar{S}^{\prime} \in|V|$ then $\pi^{-1}\left(\bar{A}^{\prime}\right)=\pi^{-1}\left[\pi\left(C_{\bar{S}}\right)\right] \subseteq \mathrm{E}$. If not, the curves $C_{S} \subseteq \mathrm{E}$ fill some surface $\pi^{-1}(Z)$ for some effective divisor $Z$ of $Y$, otherwise $\operatorname{dim}[\pi(\mathrm{E})]=2$. Hence, in any case, E contains some surface $\pi^{-1}(Z)$. By choosing $Z$ maximal we have that $|V|=\left|T+\pi^{*} B\right|+\left(\pi^{*} A-\pi^{*} B\right)$ where $B$ is a suitable divisor of $Y$ such that $T+\pi^{*} B$ is effective on $X$, where $Z=A-B$ 
is effective on $Y$ and where $\left(\pi^{*} A-\pi^{*} B\right)$ is the fixed part of $|V|$. But this is not possible, by assumption b), as $\operatorname{dim}\left(\left|T+\pi^{*} B\right|\right)=\operatorname{dim}(|V|) \geq \operatorname{dim}(|D|)-2$.

Proposition 3.1, in case $\epsilon=1$, still requires the very ampleness of a line bundle, $T_{\left.\right|_{S}}$, on a surface section $S$ of $X$. Even in the most simple situation, when $S$ is isomorphic to the blowing up of $Y$ at $c_{2}\left(\mathcal{E} \otimes \mathcal{O}_{Y}(A)\right)$ distinct points, the very ampleness of $T_{\mid S}$ depends on the position of these points on $Y$, and in general we know nothing about it. The following Proposition circumvents this difficulty by directly showing separation of length- 2 zero-schemes on $X$. The basic technique is the construction of separating elements as reducible divisors. They are constructed as sums of a horizontal component and a vertical one.

Proposition 3.5. With the notation fixed in this Section, let $B \in \operatorname{Pic}(Y)$ be effective. Assume:

a) for all pairs of distinct points $\{P, Q\} \subset X$

i) there exist $\Sigma \in\left|T+\pi^{*} A+\pi^{*} B\right|$, which is reducible as the union of a smooth surface $S_{2} \in\left|T+\pi^{*} A\right|$ passing through $P$ and not through $Q$, and a smooth surface $S_{1} \in\left|\pi^{*} B\right|$ passing through $Q$;

ii) there exist $\sigma_{1} \in H^{0}\left(T_{\mid S_{1}}\right)$, such that $\sigma_{1}(Q) \neq 0$ and $\sigma_{1}(P)=0$ in case $P \in S_{1} \cap S_{2}$

iii) there exist $\sigma_{2} \in H^{0}\left(T_{\mid S_{2}}\right)$, such that $\sigma_{2}(P)=0$;

iv) there exist $\sigma \in H^{0}\left(T_{\mid \Sigma}\right)$, such that $\sigma_{\left.\right|_{S_{1}}}=\sigma_{1}$ and $\sigma_{\left.\right|_{S_{2}}}=\sigma_{2}$;

$\beta)$ for any point $P \in X$ and for any direction $\underline{q} \in \mathbb{T}_{P}(X)$

i) there exists $\Sigma \in\left|T+\pi^{*} A+\pi^{*} B\right|$, which is reducible as the union of a smooth surface $S_{2} \in\left|T+\pi^{*} A\right|$ and a smooth surface $S_{1} \in\left|\pi^{*} B\right|$, both passing through $P$;

ii) there exists $\sigma_{1} \in H^{0}\left(T_{\mid S_{1}}\right)$ such that $\sigma_{1}(P)=0$ and $\left(\sigma_{1}\right)_{0}$ is smooth at $P$ with tangent vector $\underline{t} \in \mathbb{T}_{P}\left(S_{1}\right)$;

iii) there exists $\sigma_{2} \in H^{0}\left(T_{\mid S_{2}}\right)$ such that $\in \sigma_{2}(P)=0$ and $\left(\sigma_{2}\right)_{0}$ is smooth at $P$ with tangent vector $\underline{v} \in \mathbb{T}_{P}\left(S_{2}\right)$ in such a way that $q \notin\langle\underline{t}, \underline{v}>$;

iv) there exists $\sigma \in H^{0}\left(T_{\mid \Sigma}\right)$, such that $\sigma_{\mid S_{1}}=\sigma_{1}$ and $\sigma_{\mid S_{2}}=\sigma_{2}$.

r) $h^{1}(Y,-A-B)=0$.

Then $T$ is very ample on $X$.

Proof. For any reduced length-2 zero scheme $\{P, Q\} \in X^{[2]}$, assumption $\alpha$ ) gives a section $\sigma \in H^{0}\left(T_{\mid \Sigma}\right)$ such that $\sigma(P)=0, \sigma(Q) \neq 0$, and, reversing the roles of $P$ and $Q$, a section $\sigma^{\prime} \in H^{0}\left(T_{\mid \Sigma^{\prime}}\right)$ such that $\sigma^{\prime}(Q)=0, \sigma^{\prime}(P) \neq 0$. As in the proof of Proposition 3.1, assumption $\gamma$ ) allows such $\sigma$ and $\sigma^{\prime}$ to be lifted to elements of $H^{0}(T)$ separating $\{P, Q\}$.

For any point $P \in X$ and for any direction $\underline{q \in} \mathbb{T}_{P}(X)$, assumption $\beta$ ) gives $\sigma \in H^{0}\left(T_{\mid \Sigma}\right)$ such that $\sigma(P)=0$, and such that $\mathbb{T}_{P}\left((\sigma)_{0}\right)$ does not contain $\underline{q}$. Once again, assumption $\gamma$ ) allows $\sigma$ to be lifted to $\tau_{1} \in H^{0}(T)$ with $\tau_{1 \mid \Sigma}=\sigma$ such that $\tau_{1}(P)=0$. Notice that $\left(\tau_{1}\right)_{0}$ is smooth at $P$; in fact $\left(\tau_{1}\right)_{0}$ can be singular at $P$ only if it contains the fibre of $\pi$ through $P$, but in this case it could not cut $\left(\sigma_{1}\right)_{0}$ on $S_{1}$, as in our assumptions, because $\left(\sigma_{1}\right)_{0}$ is smooth at $P$. Hence $\mathbb{T}_{P}\left(\left(\tau_{1}\right)_{0}\right)=$ $\langle\underline{t}, \underline{v}\rangle$ and it does not contain $q$. Assumption $\alpha)$ guarantees the existence of a section $\tau_{2} \in H^{0}(T)$ such that $\tau_{2}(P) \neq 0$. Thus $\left\{\tau_{1}, \tau_{2}\right\}$ separate the zero scheme $\{P, \underline{q}\}$. 
We conclude this Section with a simple very ampleness criterion for line bundles on surfaces. The idea of this proof will be used in $\S 4$ to give a criterion for our vector bundles.

Proposition 3.6. Let $S$ be a smooth projective surface. Let $D, A \in P i c(S)$ and let $z \geq 1$ be a positive integer such that $z A$ is very ample. Assume:

1) for any $\xi \in S^{[2]}$ there exists a smooth, irreducible curve $\gamma \in|z A|$ passing through $\xi$

2) $D A \geq(z-1) A^{2}+2 p_{a}(A)+1$;

3) $h^{1}(S, D-z A)=0$.

Then $D$ is very ample.

Proof. Let $\xi \in S^{[2]}$ and let $\gamma$ be as in assumption 1). Assumption 2) implies that $D_{\mid \gamma}$ is very ample. In fact $2 g(\gamma)-2=\left(z A+K_{S}\right) z A$, hence $2 g(\gamma)+1=$ $\left(z A+K_{S}\right) z A+3$ and $\operatorname{deg}\left(D_{\mid \gamma}\right)=z D A \geq 2 g(\gamma)+1=\left(z A+K_{S}\right) z A+3$ because $D A \geq(z-1) A^{2}+2 p_{a}(A)+1=\left(z A+K_{S}\right) A+3$. As $D_{\mid \gamma}$ is very ample we have two sections $\left\{\sigma_{1}, \sigma_{1}^{\prime}\right\}$, in $H^{0}\left(\gamma, D_{\mid \gamma}\right)$, that separate $\xi$ on $\gamma$. Assumption 3) guarantees that we can separate $\xi$ on $S$ by lifting $\sigma_{1}$ and $\sigma_{1}^{\prime}$ to sections in $H^{0}(S, D)$.

Remark 3.2. The existence of certain surfaces $S \subset \mathbb{P}^{4}$ of degree 14 which are ruled over a genus 2 curve, with invariant $e=-2$, is a long standing open problem, see for example [H-R]. The candidate very ample line bundle giving the embedding of $S$ is predicted to be of numerical class $7 C_{0}-6 f$. As an application of Proposition 3.6 one can prove that $D \equiv 7 C_{0}+2 f$ is very ample on $S$, by setting $A \equiv C_{0}+5 f$, and $z=1$. One could then try to solve the original problem by embedding $S$ with $D$ and finding a suitable projection.

\section{A NUMERICAL VERY AMPLENESS CRITERION}

As indicated in the introduction, when the base surface $Y$ has a simple Picard group, one can hope to obtain sufficient conditions to characterize very ampleness of vector bundles over $Y$.

The heart of this section is Theorem 4.2. It is obtained using the same approach described in Section 3 with an interesting twist. The divisor $D$, chosen through the length 2 zero-scheme that needs separation, is obtained as a sum of a number of components, all chosen as pull backs of suitable sections of the base surface. Similarly, the global sections of the tautological line bundle $T$, performing the required separation are lifted from sections glued together from well behaved sections on each of the components of $D$.

Firstly we have the following

Lemma 4.1. Let $Y=\mathbf{F}_{e}$ and let $A \equiv C_{0}+$ bf be a very ample divisor on $Y$. Let $P_{1}, \ldots, P_{r}$ be $r$ distinct points on $Y$ such that no two of them lie on the same fibre. Let $F:=\left\{f_{i}\right\}$ where $f_{i}=\rho^{*}\left(\rho\left(P_{i}\right)\right.$. If $h^{0}(Y, A) \geq 2 r$ then there exists at least an element $\gamma \in|A|$ passing smoothly through $P_{1}, \ldots, P_{r}$ and intersecting transversely any fixed finite set of fibres $\Phi:=\left\{\varphi_{j}\right\}$ with $\Phi \cap F=\emptyset$.

Proof. First of all, let us consider the case in which the set $\Phi$ is empty. For any point $P_{i}$ let us choose a vector $q_{i} \in \mathbb{T}_{P_{i}}(Y)$ pointing towards a direction different from that of $f_{i}$. By assumption, there exists at least an element $\gamma \in|A|$ passing through $P_{1}, \ldots, P_{r}$ and having tangent direction $\underline{q}_{i}$ at $P_{i} i=1, \ldots, r$ ( $2 r$ linear conditions $)$. 
If $\gamma$ is singular at, say, $P_{1}, \ldots, P_{h}$ then $\gamma$ must be reducible into an element $\gamma^{\prime} \in$ $\left|A-b_{1} f_{1}-b_{2} f_{2}-\ldots-b_{h} f_{h}\right|$ and the union of fibres $f_{1}, f_{2}, \ldots, f_{h}$ with multiplicities $b_{1}, b_{2}, \ldots, b_{h}$ (see also the proof of Proposition 3.4). Now $\gamma^{\prime}$ is smooth at any $P_{i}$ and, by our assumptions, $\gamma^{\prime}$ has tangent vector $q_{i}$ at any $P_{i}$. One can choose a set of $h$ generic fibres $\left\{f_{1}^{\prime}, f_{2}^{\prime}, \ldots, f_{h}^{\prime}\right\} \cap F=\emptyset$ so that $\gamma^{\prime}+b_{1} f_{1}^{\prime}+b_{2} f_{2}^{\prime}+\ldots+b_{h} f_{h}^{\prime} \in|A|$ satisfies our request; recall that $b_{1} f_{1}^{\prime}+b_{2} f_{2}^{\prime}+\ldots+b_{h} f_{h}^{\prime}$ is linearly equivalent to $b_{1} f_{1}+b_{2} f_{2}+\ldots+b_{h} f_{h}$.

Now let us assume that $\Phi$ is not empty and let us proceed as in the previous case. If $\gamma^{\prime}$ cuts every fibre of $\Phi$ tranversally we are done. Otherwise $\gamma^{\prime}$ must be reducible into an element $\gamma^{\prime \prime} \in\left|A-b_{1} \varphi_{1}-b_{2} \varphi_{2}-\ldots-b_{h} \varphi_{h}\right|$ and the union of fibres $\varphi_{1}, \varphi_{2}, \ldots, \varphi_{h}$ with multiplicities $b_{1}, b_{2}, \ldots, b_{h}$. One can then argue as in the previous case.

Definition 4.1. Let $W$ be a 0-dimensional reduced scheme on a ruled surface $Y$. For any fixed fibre $\bar{f}$ of $Y$ we can compute length $\left(\mathcal{O}_{Y}(\bar{f}) \otimes \mathcal{O}_{W}\right)$. Let $\operatorname{lm}_{Y}(W)$ be the maximun of these lengths as $\bar{f}$ varies among the fibre of $Y$.

We can now prove prove the main Theorem of this section.

Theorem 4.2. Let $Y=\mathbf{F}_{e}$ and let $L=a_{l} C_{0}+b_{l} f$ and $M=a_{m} C_{0}+b_{m} f$ be line bundles over $Y$. Let $\mathcal{E}$ be a rank 2 vector bundle over $Y$ such that there exists a non trivial exact sequence $0 \rightarrow L \rightarrow \mathcal{E} \rightarrow M \otimes \mathcal{I}_{W} \rightarrow 0$ where $\mathcal{I}_{W}$ is the ideal sheaf of a 0 -dimensional reduced subscheme $W \subset Y$ of length $w$. Let $X=\mathbb{P}(\mathcal{E})$ and let $T$ be the tautological line bundle on $X$.

Assume that there exist integers $x \geq e+2$ and $z \geq 1$ such that:

1) $L\left(C_{0}+x f\right)>0, M\left(C_{0}+x f\right)>2$

2) $L f>0, M f>\operatorname{lm}_{Y}(W)$

3) $h^{1}\left(Y, L-z C_{0}-z x f\right)=0, h^{1}\left(Y, M-z C_{0}-z x f\right)=0$

4) $h^{1}\left(Y, L-z C_{0}-z x f-f\right)=0, h^{1}\left(Y, M-z C_{0}-z x f-f\right)=0$

$5)$ the support of $W$ is in general position with respect to the linear systems $\left|M-z\left(C_{0}+x f\right)\right|$ and $\left|M-z\left(C_{0}+x f\right)-f\right|$ (i.e. the following natural maps $H^{0}\left(Y, M-z\left(C_{0}+x f\right)\right) \rightarrow H^{0}\left(Y,\left(M-z\left(C_{0}+x f\right)\right) \otimes \mathcal{O}_{W}\right)$ and $H^{0}\left(Y, M-z\left(C_{0}+\right.\right.$ $x f)-f) \rightarrow H^{0}\left(Y,\left(M-z\left(C_{0}+x f\right)-f\right) \otimes \mathcal{O}_{W}\right)$ are surjective $)$

6) $(L+M)\left(C_{0}+x f\right) \geq 2(z-1)(2 x-e)$.

Then $T$ is very ample.

Proof. Let us consider the linear system $\left|C_{0}+x f\right|$ on $Y$ and notice that it is very ample by the assumption $x \geq e+2$ and [H], pag.169. Moreover there are no $\left|C_{0}+x f\right|$-lines by Lemma 2.1 (other than fibres). Let $\gamma \in\left|C_{0}+x f\right|$ be a smooth rational curve passing through at most 2 points of $W$. Let us consider the restriction $\mathcal{E}_{\mid \gamma}$. By assumption 1) $\mathcal{E}_{\mid \gamma}$ is ample, hence very ample because $\gamma$ is a smooth rational curve.

A similar argument shows that, for any fibre $f$ of the ruling of $Y$, the restriction $\mathcal{E}_{\mid f}$ is also very ample, by assumption 2 ).

Let us consider the following exact sequences:

$0 \rightarrow L-z \gamma \rightarrow \mathcal{E} \otimes \mathcal{O}_{Y}(-z \gamma) \rightarrow(M-z \gamma) \otimes \mathcal{I}_{W} \rightarrow 0$

$0 \rightarrow(M-z \gamma) \otimes \mathcal{I}_{W} \rightarrow M-z \gamma \rightarrow(M-z \gamma) \otimes \mathcal{O}_{W} \rightarrow 0$.

By using assumptions 3) and 5) it is easy to see that $h^{1}\left(Y, \mathcal{E} \otimes \mathcal{O}_{Y}(-z \gamma)\right)=0$.

Let us consider the following exact sequences:

$0 \rightarrow L-z \gamma-f \rightarrow \mathcal{E} \otimes \mathcal{O}_{Y}(-z \gamma-f) \rightarrow(M-z \gamma-f) \otimes \mathcal{I}_{W} \rightarrow 0,0 \rightarrow(M-z \gamma-$ $f) \otimes \mathcal{I}_{W} \rightarrow M-z \gamma-f \rightarrow(M-z \gamma-f) \otimes \mathcal{O}_{W} \rightarrow 0$ 
By using assumptions 4$)$ and 5$)$ it is easy to see that $h^{1}\left(Y, \mathcal{E} \otimes \mathcal{O}_{Y}(-z \gamma-f)\right)=0$. Given a divisor $D \equiv z \pi^{*} \gamma$ on $X$ and the exact sequence $0 \rightarrow T-D \rightarrow T \rightarrow T \otimes \mathcal{O}_{D} \rightarrow 0$, the above vanishings give that the natural map

$$
|T| \rightarrow\left|T_{\mid D}\right| \rightarrow 0
$$

is surjective. A similar argument shows that if $D \equiv z \pi^{*} \gamma+\pi^{*} f$, the map in (6) is surjective too.

To show that $T$ is very ample we have to prove that $|T|$ separates all zero schemes $\xi \subset X^{[2]}$. The proof is divided into two cases, according to the nature of $\xi$. When $\xi$ is not reduced, and $\operatorname{Supp}(\xi)=\{P\}$, we denote by $\underline{q} \in \mathbb{T}_{P}(X)$ the tangent direction specified by $\xi$.

Case 1: $P \in \operatorname{Supp}(\xi)$ and $\xi \nsubseteq F$ (scheme theoretically), where $F=\mathbb{P}\left(\mathcal{E}_{\mid f}\right)$ and $f=\rho^{-1}(\rho(\pi(P))$.

If $\operatorname{Supp}(\xi)=\{P, Q\}$, as $\left|C_{0}+x f\right|$ is very ample, [B-S] Theorem 1.7.9, and Lemma 2.1 imply that there exists a smooth rational curve $\gamma_{1} \in\left|C_{0}+x f\right|$ containing $\pi(P)$ and $\pi(Q)$. If $\xi$ is not reduced, again very ampleness of $\left|C_{0}+x f\right|$, [B-S] Theorem 1.7.9, and Lemma 2.1 imply that there exists a smooth rational curve $\gamma_{1} \in\left|C_{0}+x f\right|$ passing through $\pi(P)$, with $q \in \mathbb{T}_{P}\left(\Gamma_{1}\right)$, where $\Gamma_{1}=\mathbb{P}\left(\mathcal{E}_{\mid \gamma_{1}}\right)$. By choosing suitably $\gamma_{1}$ we can assume that $\gamma_{1}$ contains at most two of the points of $W(\pi(P)$ and $\pi(Q)$ if it is the case): recall that there are no curves, other than fibres, embedded as lines by $\left|C_{0}+x f\right|$ thanks to Lemma 2.1. Choose another smooth rational curve $\gamma_{2} \in\left|C_{0}+x f\right|$ such that $\gamma_{2}$ intersects $\gamma_{1}$ transversely at $\left(C_{0}+x f\right)^{2}=2 x-e$ points all different from $\pi(\operatorname{Supp}(\xi))$ and not belonging to $W$. Now choose another smooth rational curve $\gamma_{3} \in\left|C_{0}+x f\right|$ such that $\gamma_{3}$ and $\Gamma_{3}$ have the same above properties with respect to $\gamma_{1}, \gamma_{2}, \Gamma_{1}, \Gamma_{2}, W$ and moreover $\bigcap_{i=1}^{3} \gamma_{i}=\emptyset$. Iterate the process, until $i=z$. Note that the suitable choice of the $\gamma_{i}$ is possible simply because $\left|C_{0}+x f\right|$ is very ample on $Y$.

Let $\Gamma_{i}$ also denote the numerical classes of $\Gamma_{i}$ in $\operatorname{Num}(X)$. If $z=1$ we can separate $\xi$ on $\Gamma_{1}$ because $T_{\mid \Gamma_{1}}$ is very ample, then we are done by lifting the separating sections with the map in (6). If $z \geq 2$ we need to apply Lemma 4.1 to $T_{\mid \Gamma_{i}}$ when $i \geq 2$. Let us remark that $h^{0}\left(\Gamma_{i}, T_{\mid \Gamma_{i}}\right)=h^{0}\left(\Gamma_{2}, T_{\mid \Gamma_{2}}\right)=(L+M) \gamma_{2}+2=$ $(L+M)\left(C_{0}+x f\right)+2$ for $i=2, \ldots, z$, so that assumption 6$)$ implies that $h^{0}\left(\Gamma_{i}, T_{\mid \Gamma_{i}}\right) \geq$ $2(z-1)(2 x-e)$ for any $i=2, \ldots, z$. Noticing that $\Gamma_{i} \cap \Gamma_{j}$ is the disjoint union of $2 x-e$ fibres of $\pi$, we can proceed as follows: $T_{\mid \Gamma_{1}}$ is very ample, so we can take $s_{1}^{1} \in\left|T_{\mid \Gamma_{1}}\right|$ and $s_{1}^{2} \in\left|T_{\mid \Gamma_{1}}\right|$ separating $\xi$ on $\Gamma_{1}$. We can also choose $s_{1}^{1}$ and $s_{1}^{2}$ such that they intersect transversely all the fibres $\Gamma_{1} \cap \Gamma_{j} j=2, \ldots, z$. As $h^{0}\left(\Gamma_{2}, T_{\mid \Gamma_{2}}\right) \geq 2(2 x-e)$, by Lemma 4.1, we can choose $s_{2}^{1} \in\left|T_{\mid \Gamma_{2}}\right|$ and $s_{2}^{2} \in\left|T_{\mid \Gamma_{2}}\right|$ such that $s_{1}^{k} \cap \Gamma_{2}=s_{2}^{k} \cap \Gamma_{1}$, for $k=1,2$, and such that they intersect transversely all the fibres $\Gamma_{2} \cap \Gamma_{j} j=3, \ldots, z$. As $h^{0}\left(\Gamma_{3}, T_{\mid \Gamma_{3}}\right) \geq 4(2 x-e)$, by Lemma 4.1 , we can choose $s_{3}^{1} \in\left|T_{\mid \Gamma_{3}}\right|$ and $s_{3}^{2} \in\left|T_{\mid \Gamma_{3}}\right|$ such that $s_{3}^{k} \cap \Gamma_{2}=s_{2}^{k} \cap \Gamma_{3}$, and $s_{3}^{k} \cap \Gamma_{1}=s_{1}^{k} \cap \Gamma_{3}$ for $k=1,2$, and such that they intersect transversely all the fibres $\Gamma_{3} \cap \Gamma_{j} j=4, \ldots, z$. And so on. At the end we get, for each $k=1,2$, a set of $z$ elements $s_{i}^{k} \in\left|T_{\mid \Gamma_{i}}\right| i=1, . ., z$ such that for any $i, j \in\{1, \ldots, z\}, i \neq j, s_{i}^{k} \cap \Gamma_{j}=s_{j}^{k} \cap \Gamma_{i}$ is a reduced zero-subscheme, and $\left\{s_{1}^{1}, s_{1}^{2}\right\}$ separate $\xi$ on $\Gamma_{1}$. Thus, for each $k=1,2$, the $z$-tuples $\left\{s_{i}^{k} \in\left|T_{\mid \Gamma_{i}}\right| i=1, \ldots, z\right\}$ give rise to elements $\bar{s}^{k}$ of $\left|T_{\mid\left(\Gamma_{1} \cup \ldots \cup \Gamma_{z}\right)}\right|=\left|T_{\mid\left(\Gamma_{1}+\ldots+\Gamma_{z}\right)}\right|$ which still separate $\xi$ on $\Gamma_{1} \cup \ldots \cup \Gamma_{z}$. As $\Gamma_{1}+\ldots+\Gamma_{z} \equiv z \pi^{*} \gamma$, (6) gives elements $s^{k}$ of $|T|$ that separate $\xi$ on $X$. 
Case 2: $P \in \operatorname{Supp}(\xi)$ and $\xi \subset F$ (scheme theoretically), where $F=\mathbb{P}\left(\mathcal{E}_{\mid f}\right)$ and $f=\rho^{-1}(\rho(\pi(P))$.

Let $F$ also denote the numerical class of $\mathbb{P}\left(\mathcal{E}_{\mid f}\right)$ in $\operatorname{Num}(X)$. As in the previous case, let us choose $z$ smooth rational curves $\gamma_{1}, \ldots, \gamma_{z} \in\left|C_{0}+x f\right|$ such that they intersect each other transversely at $\left(C_{0}+x f\right)^{2}=2 x-e$ points, each of them intersects $f$ transversely at one point different from $\pi(\operatorname{Supp}(\xi))$, not belonging to $W$, and such that any 3 -tuple of curves in $\left\{\gamma_{1}, \ldots, \gamma_{z}, f\right\}$ has empty intersection. Note that all the suitables choices ar possible because $\left|C_{0}+x f\right|$ is very ample.

Let $\Gamma_{i}$ also denote the numerical class of $\Gamma_{i}=\mathbb{P}\left(\mathcal{E}_{\mid \gamma_{i}}\right)$ in $\operatorname{Num}(X)$. We get the same results about $h^{0}\left(\Gamma_{i}, T_{\mid \Gamma_{i}}\right)$ as in Case 1 and we have $h^{0}\left(\Gamma_{i}, T_{\mid \Gamma_{i}}\right)=(L+M)\left(C_{0}+\right.$ $x f)+2$ for any $i=1, \ldots, z$.

As $T_{\mid F}$ is very ample we can take $s_{0}^{1} \in\left|T_{\mid F}\right|$ and $s_{0}^{2} \in\left|T_{\mid F}\right|$ separating $\xi$ on $F$. We can also choose $s_{0}^{1}$ and $s_{0}^{2}$ such that they intersect transversely all the fibres $F \cap \Gamma_{j} j=1, \ldots, z$. Then we can choose $s_{1}^{1} \in\left|T_{\mid \Gamma_{1}}\right|$ and $s_{1}^{2} \in\left|T_{\mid \Gamma_{1}}\right|$ such that $s_{1}^{k} \cap F=s_{0}^{k} \cap \Gamma_{1}$, for $k=1,2$, and such that they intersect transversely all the fibres $\Gamma_{1} \cap \Gamma_{j} j=2, \ldots, z$. As $h^{0}\left(\Gamma_{2}, T_{\mid \Gamma_{2}}\right) \geq 2[(2 x-e)+1]$, by Lemma 4.1, we can choose $s_{2}^{1} \in\left|T_{\mid \Gamma_{2}}\right|$ and $s_{2}^{2} \in\left|T_{\mid \Gamma_{2}}\right|$ such that $s_{1}^{k} \cap \Gamma_{2}=s_{2}^{k} \cap \Gamma_{1}, s_{2}^{k} \cap F=s_{0}^{k} \cap \Gamma_{2}$ for $k=1,2$, and such that they intersect transversely all the fibres $\Gamma_{3} \cap \Gamma_{j} j=3, \ldots, z$. and so on. At the end, by recalling that assumption 6$)$ gives $h^{0}\left(\Gamma_{i}, T_{\mid \Gamma_{i}}\right) \geq 2[(z-1)(2 x-e)+1]$, for any $i=1, \ldots, z$, we get a $(z+1)$-tuples $\left\{s_{i}^{k}, i=0,1, \ldots, z\right\}$ for $k=1,2$, giving rise to two elements $\bar{s}^{k} \in\left|T_{\mid\left(F \cup \Gamma_{1} \cup \ldots \cup \Gamma_{z}\right)}\right|=\left|T_{\mid\left(F+\Gamma_{1}+\ldots+\Gamma_{z}\right.}\right|$ that separate $\xi$ on $F \cup \Gamma_{1} \cup \ldots \cup \Gamma_{z}$. As $\Gamma_{1}+\ldots+\Gamma_{z}+F \equiv z \pi^{*} \gamma+\pi^{*} f,(6)$ gives elements $s^{k} \in|T|$ that separate $\xi$ on $X$.

Remark 4.1. Note that the previous criterion is purely numerical because condition 5) can be translated into a vanishing condition as we have seen in the proof of Theorem 4.2.

To show the large validity of Theorem 4.2 we give the following example.

Example: let us consider on $Y=\mathbf{F}_{e}$ two line bundles $L \equiv a C_{0}+b_{l} f$ and $M \equiv(a+2) C_{0}+b_{m} f$ with $a>0$. Let us consider $K_{Y} \equiv-2 C_{0}-(2+e) f$ so that $K_{Y}+M-L \equiv\left(b_{m}-b_{l}-e-2\right) f$. Let us assume $b_{m}-b_{l}-e-2>0$ and let us choose $W=\{$ two distinct points on a fixed fibre $\bar{f}\}$, hence $w=2$. In this way we can apply Griffiths-Harris theorem on the existence of rank 2 vector bundles on surfaces (because every section of $\left|K_{Y}+M-L\right|$ passing through a point of $W$, passes also through the other point, see [D-L], Theorem 3.13 and its proof) and we get an exact sequence as the following: $0 \rightarrow \mathcal{O}_{Y} \rightarrow \mathcal{E}^{\prime} \rightarrow(M-L) \otimes \mathcal{I}_{W} \rightarrow 0$.

By tensorizing with $L$ we get our vector bundle $\mathcal{E}$ :

$$
0 \rightarrow L \rightarrow \mathcal{E} \rightarrow M \otimes \mathcal{I}_{W} \rightarrow 0 .
$$

Let us fix $x=e+2$ and $z=a+1$ and let us write down all necessary numerical conditions to satisfy the assumptions of Theorem 4.2.

1) $2 a+b_{l}>0$ and $2 a+4+b_{m}>2$.

2) $a>0$.

3) $b_{m}-(a+1)(e+2) \geq e-1$.

4) $b_{m}-(a+1)(e+2)-1 \geq e-1$.

5) $b_{m}-(a+1)(e+2)-1 \geq e$ and $b_{m}-(a+1)(e+2)-2 \geq e$.

6) $b_{l}+b_{m} \geq 4 a+2 a e-4$.

By looking at all required conditions in theorem 4.2 one can show that, for fixed $e, a>0, b_{l}>-2 a$, all assumptions are satisfied for $b_{m}>>0$, thus obtaining the very 
ampleness of a large class vector bundles $\mathcal{E}$. These bundles give rise to 3 -folds scrolls embedded with fairly large degree (see Remark 3.1 and Section 7). For instance, if $d:=\operatorname{deg}(X)$, we have $d=c_{1}(\mathcal{E})^{2}-c_{2}(\mathcal{E})=(3 a+4)\left(b_{m}+b_{l}\right)-2 b_{l}-e\left(3 a^{2}+6 a+4\right)-2$, and, if we put $e=a=1, b_{l}=-1, b_{m}=9$, we get $d=43$.

\section{A CRITERION FOR RULED SURFACES ON ELLIPTIC CURVES}

In this section we want to give some very ampleness criterion when the surface $Y$ is a ruled surface on an elliptic curve, i.e. $Y=\mathbb{P}(\mathcal{F})$, where $\mathcal{F}$ is a normalized rank 2 vector bundle over a smooth elliptic curve $C$ with invariant $e$. Normalized means that $h^{0}(C, \mathcal{F}) \neq 0$, but $h^{0}(C, \mathcal{F} \otimes \mathcal{L})=0$ for any linear bundle $\mathcal{L}$ on $C$ of negative degree. As usual, let $\rho: Y \rightarrow C$ be the natural map.

To get our criterion, in this case we will need stronger assumptions. Let us begin with the following.

Lemma 5.1. Let $Y$ be an elliptic ruled surface as above over an elliptic curve $C$. Let $A \equiv C_{0}+$ bf a very ample divisor on $Y$ (where $C_{0}$ and $f$ are, respectively, the numerical classes of the tautological line bundle and of the fibre). Let $P_{1}, \ldots, P_{r}$ be $r \geq 2$ distinct points on $Y$ such that no two of them lie on the same fibre. Let $F:=$ $\left\{f_{P_{1}}, f_{P_{2}}, \ldots, f_{P_{r}}\right\}$ where $f_{P_{i}}=\rho^{*} \rho\left(P_{i}\right)$. If $h^{0}(Y, A) \geq 2 r+2$ then there exists at least an element $\gamma \in|A|$ passing smoothly through $P_{1}, \ldots, P_{r}$, intersecting any $f_{P_{i}}$ tranversely and intersecting transversely any other finite set of fibres $\Phi:=\left\{\varphi_{j}\right\}$ with $F \cap \Phi=\emptyset$.

Proof. Firstly, let us assume that $\Phi$ is empty. For any point $P_{i}$ let us choose a vector $\underline{q}_{i} \in \mathbb{T}_{P_{i}}(Y)$ pointing towards a direction different from that of $f_{P_{i}}$. By assumption, there exists at least a pencil of elements $\gamma \in|A|$ passing through $P_{1}, \ldots, P_{r}$ and having tangent direction $q_{i}$ at $P_{i} i=1, \ldots, r$ ( $2 r$ linear conditions). If there exists at least an element $\gamma \in|A|$ passing smoothly through $P_{1}, \ldots, P_{r}$ and cutting any $f_{P_{i}}$ tranversally we are done. Otherwise every $\gamma$ is singular at some points, say, $P_{1}, \ldots, P_{s}$ and therefore every $\gamma$ must be reducible into an element $\gamma^{\prime} \in\left|A-b_{1} f_{P_{1}}-b_{2} f_{P_{2}}-\ldots-b_{s} f_{P_{s}}\right|$ and the union of fibres $f_{P_{1}}, f_{P_{2}}, \ldots, f_{P_{s}}$ with multiplicities $b_{1}, b_{2}, \ldots, b_{s}$ (see also the proof of Proposition 3.4). Now the generic $\gamma^{\prime}$ is smooth at any $P_{i}$ and, by our assumptions, $\gamma^{\prime}$ has tangent vector $\underline{q}_{i}$ at any $P_{i}$.

Let $\rho: Y \rightarrow C$ be the natural projection. If $b:=b_{1}+b_{2}+\ldots+b_{s} \geq 3$, we can choose other $b$ generic points $H_{1}, \ldots, H_{b} \in C$ (not necessarily distinct) such that $f_{H_{1}}+f_{H_{2}}+\ldots+f_{H_{b}}$ is linearly equivalent to $b_{1} f_{P_{1}}+b_{2} f_{P_{2}}+\ldots+b_{s} f_{P_{s}}$. This is possible because $b_{1} \rho\left(P_{1}\right)+b_{2} \rho\left(P_{2}\right)+\ldots+b_{s} \rho\left(P_{s}\right)$ is a very ample divisor on $C$, so that it suffices to choose a generic divisor $H_{1}+\ldots+H_{b}$ (disjoint with $\left.b_{1} \rho\left(P_{1}\right)+b_{2} \rho\left(P_{2}\right)+\ldots+b_{s} \rho\left(P_{s}\right)\right)$ in the linear system $\left|b_{1} \rho\left(P_{1}\right)+b_{2} \rho\left(P_{2}\right)+\ldots+b_{s} \rho\left(P_{s}\right)\right|$. Now we can consider an element $\gamma^{\prime}+f_{H_{1}}+f_{H_{2}}+\ldots+f_{H_{b}} \in|A|$ and we are done.

If $b:=b_{1}+b_{2}+\ldots+b_{s}=2$ we can choose other 2 generic points $H_{1}, H_{2} \in C$ (not necessarily distinct) such that $f_{H_{1}}+f_{H_{2}}$ is linearly equivalent to $b_{1} f_{P_{1}}+b_{2} f_{P_{2}}+$ $\ldots+b_{s} f_{P_{s}}$. This is possible because $b_{1} \rho\left(P_{1}\right)+b_{2} \rho\left(P_{2}\right)+\ldots+b_{s} \rho\left(P_{s}\right)$ is a degree 2 effective divisor on $C$, hence $\left|b_{1} \rho\left(P_{1}\right)+b_{2} \rho\left(P_{2}\right)+\ldots+b_{s} \rho\left(P_{s}\right)\right|$ is a one dimensional linear system without base points and it suffices to choose a generic divisor $H_{1}+H_{2}$ (disjoint with $b_{1} \rho\left(P_{1}\right)+b_{2} \rho\left(P_{2}\right)+\ldots+b_{s} \rho\left(P_{s}\right)$ ) in it. Now we can consider an element $\gamma^{\prime}+f_{H_{1}}+f_{H_{2}} \in|A|$ and we are done.

If $b:=b_{1}+b_{2}+\ldots+b_{s}=1$, say $b_{1}=1$ and $b_{2}=\ldots=b_{s}=0$, every $\gamma$ must be reducible into an element $\gamma^{\prime} \in\left|A-f_{P_{1}}\right|$ and the fibre $f_{P_{1}}$. Because there exists 
at least a pencil of elements $\gamma \in|A|$ passing through $P_{1}, \ldots, P_{r}$ and having tangent direction $q_{i}$ at $P_{i} i=1, \ldots, r$, we can choose another point $P^{\prime} \in f_{P_{2}}, P^{\prime} \neq P_{2}$ (recall that $r \geq 2$ ) and we can find at least an element $\bar{\gamma} \in|A|$ passing through $P_{1}, \ldots, P_{r}, P^{\prime}$ and having tangent direction $\underline{q}_{i}$ at $P_{i} i=1, \ldots, r$. We have that $\bar{\gamma}$ is reducible into an element $\overline{\gamma^{\prime}} \in\left|A-f_{P_{1}}-f_{P_{2}}\right|$ and the two fibres $f_{P_{1}}, f_{P_{2}}$. As above, $\overline{\gamma^{\prime}}$ is smooth at any $P_{i}$ and, by our assumptions, $\overline{\gamma^{\prime}}$ has tangent vector $\underline{q}_{i}$ at any $P_{i}$, moreover, as above, we can choose a generic divisor $H_{1}+H_{2}$ (disjoint with $\left.\rho\left(P_{1}\right)+\rho\left(P_{2}\right)\right)$ such that $f_{H_{1}}+f_{H_{2}}$ is linearly equivalent to $f_{P_{1}}+f_{P_{2}}$. Now we can consider the element $\overline{\gamma^{\prime}}+f_{H_{1}}+f_{H_{2}} \in|A|$ and we are done.

Now let us assume that $\Phi$ is not empty, with $j=1,2, \ldots, p$, and let us proceed as in the previous case. If $\gamma^{\prime}$ (or $\overline{\gamma^{\prime}}$ ) intersects every fibre of $\Phi$ tranversally we are done. Otherwise $\gamma^{\prime}$ (or $\overline{\gamma^{\prime}}$ ) must be reducible into an element $\gamma^{\prime \prime} \in \mid A-b_{1} \varphi_{1}-$ $b_{2} \varphi_{2}-\ldots-b_{p} \varphi_{p} \mid$ and the union of fibres $\varphi_{1}, \varphi_{2}, \ldots, \varphi_{p}$ with multiplicities $b_{1}, b_{2}, \ldots, b_{p}$. One can then argue as in the previous case. Note that, as all our choices are made by using generic points $H_{i}$ on $C$, we can avoid any fixed set of points on $C$.

Now let us return to the surface $Y=\mathbb{P}(\mathcal{F})$. It is well known that, if $\mathcal{F}$ is indecomposable, hence semistable, then $0 \leq-e:=\operatorname{deg}(\mathcal{F})=\operatorname{deg}\left[c_{1}(\mathcal{F})\right] \leq 1$; if $\mathcal{F}$ is decomposable then $\mathcal{F}=\mathcal{O}_{C} \oplus \overline{\mathcal{L}}$ where $\overline{\mathcal{L}}$ is a line bundle of degree $-e \leq 0$; $($ see $[\mathrm{H}]$, $\mathrm{V}$, Theorem 2.12 and 2.15). Moreover if we compute the invariant $\mu^{-}(\mathcal{F})$ (see [Bu] for the definition) we have that $\mu^{-}(\mathcal{F})=-\frac{e}{2}$ in the first case and $\mu^{-}(\mathcal{F})=-e$ in the second case.

We have the following version of Theorem 4.2 for elliptic ruled surfaces:

Theorem 5.2. Let $Y=\mathbb{P}(\mathcal{F})$ be a surface as above. Let $L \equiv a_{l} C_{0}+b_{l} f, M \equiv$ $a_{m} C_{0}+b_{m} f$ be line bundles over $Y$. Let $\mathcal{E}$ be a rank 2 vector bundle over $Y$ such that there exists an exact sequence $0 \rightarrow L \rightarrow \mathcal{E} \rightarrow M \otimes \mathcal{I}_{W} \rightarrow 0$ where $\mathcal{I}_{W}$ is the ideal sheaf of a 0 -dimensional reduced scheme $W \subset Y$ of length $w$. Let $X=\mathbb{P}(\mathcal{E})$ and let $T$ be the tautological line bundle on $X$.

Assume that there exist integers $x$ and $z \geq 1$ such that:

0) $x+\mu^{-}(\mathcal{F}) \geq 3\left(\right.$ or $x+\mu^{-}(\mathcal{F})>1$ if $\mathcal{F}$ has degree 1$)$

1) $\min \left\{x a_{l}+b_{l}-a_{l} e, x a_{m}+b_{m}-a_{m} e-2\right\} \geq 3$

2) $L f>0, M f>\operatorname{lm}_{Y}(W)$

3) $h^{1}\left(Y, L-z C_{0}-z x f\right)=0, h^{1}\left(Y, M-z C_{0}-z x f\right)=0$

4) $h^{1}\left(Y, L-z C_{0}-z x f-f\right)=0, h^{1}\left(Y, M-z C_{0}-z x f-f\right)=0$

5) the support of $W$ is in general position with respect to the linear systems $\left|M-z\left(C_{0}+x f\right)\right|$ and $\left|M-z\left(C_{0}+x f\right)-\rho^{*} P\right|$ (i.e. the following natural maps $H^{0}\left(Y, M-z\left(C_{0}+x f\right)\right) \rightarrow H^{0}\left(Y,\left(M-z\left(C_{0}+x f\right)\right) \otimes \mathcal{O}_{W}\right)$ and $H^{0}\left(Y, M-z\left(C_{0}+\right.\right.$ $\left.x f)-\rho^{*} P\right) \rightarrow H^{0}\left(Y,\left(M-z\left(C_{0}+x f\right)-\rho^{*} P\right) \otimes \mathcal{O}_{W}\right)$ are surjective $)$, where $P$ is any point of $C$

6) $\left(a_{l}+a_{m}\right)(x-e)+b_{l}+b_{m} \geq 2(z-1)[(2 x-e)+1]+2$.

Then $T$ is very ample.

Proof. Let us recall (see $[\mathrm{M}]$ ) that a divisor on $Y$, whose numerical class is $\alpha C_{0}+\beta f$, is ample if and only if $\alpha \geq 1, \beta+\alpha \mu^{-}(\mathcal{F})>0$. The divisor is very ample if $\beta+\alpha \mu^{-}(\mathcal{F}) \geq 3$ or $\beta+\alpha \mu^{-}(\mathcal{F})>1$ if $\mathcal{F}$ has degree 1 (see $[\mathrm{A}-\mathrm{B}-\mathrm{B}]$ ).

Let us consider a linear system $\left|C_{0}+\rho^{*} \varkappa\right|$ with $\operatorname{deg}(\varkappa):=x$. By 0$)$ it is very ample. Let $\gamma$ be any smooth elliptic curve in this system passing through at most 2 points of $W$. Let us consider the restriction $\mathcal{E}_{\mid \gamma}$. We get an exact sequence: $0 \rightarrow \mathcal{L} \rightarrow$ $\mathcal{E}_{\mid \gamma} \rightarrow \mathcal{M} \rightarrow 0$ where $\mathcal{L}$ and $\mathcal{M}$ are linear bundles on $\gamma$ of degrees $x a_{l}+b_{l}-a_{l} e+\varepsilon$ 
and $x a_{m}+b_{m}-a_{m} e-\varepsilon$ respectively, and $\varepsilon=0,1,2$ is the number of common points among $\gamma$ and $W$. Recall that $\mu^{-}\left(\mathcal{E}_{\mid \gamma}\right) \geq \min \left\{x a_{l}+b_{l}-a_{l} e+\varepsilon, x a_{m}+b_{m}-a_{m} e-\varepsilon\right\}$ (see $[\mathrm{Bu}])$. By 1) $\mu^{-}\left(\mathcal{E}_{\mid \gamma}\right) \geq 3$, so that $\mathcal{E}_{\mid \gamma}$ is very ample by Theorem 3.3 and Proposition 3.2 of $[\mathrm{A}-\mathrm{B}-\mathrm{B}]$.

If we fix any fibre $f$ of $Y$ and we consider $\mathcal{E}_{\mid f}$, we get that $\mathcal{E}_{\mid f}$ is isomorphic to $\mathcal{O}_{\mathbb{P}^{1}}(h) \oplus \mathcal{O}_{\mathbb{P}^{1}}(k)$, with $h, k>0$ by assumption 2$)$, so that $\mathcal{E}_{\mid f}$ is very ample.

Let us consider the following exact sequences:

$0 \rightarrow L-z \gamma \rightarrow \mathcal{E} \otimes \mathcal{O}_{Y}(-z \gamma) \rightarrow(M-z \gamma) \otimes \mathcal{I}_{W} \rightarrow 0$

$0 \rightarrow(M-z \gamma) \otimes \mathcal{I}_{W} \rightarrow M-z \gamma \rightarrow(M-z \gamma) \otimes \mathcal{O}_{W} \rightarrow 0$.

By using assumptions 3) and 5) it is easy to see that $h^{1}\left(Y, \mathcal{E} \otimes \mathcal{O}_{Y}(-z \gamma)\right)=0$.

Let us consider the following exact sequences, for any point $P \in C$ :

$0 \rightarrow L-z \gamma-\rho^{*} P \rightarrow \mathcal{E} \otimes \mathcal{O}_{Y}\left(-z \gamma-\rho^{*} P\right) \rightarrow\left(M-z \gamma-\rho^{*} P\right) \otimes \mathcal{I}_{W} \rightarrow 0$,

$0 \rightarrow\left(M-z \gamma-\rho^{*} P\right) \otimes \mathcal{I}_{W} \rightarrow M-z \gamma-\rho^{*} P \rightarrow\left(M-z \gamma-\rho^{*} P\right) \otimes \mathcal{O}_{W} \rightarrow 0$.

By using assumptions 4$)$ and 5$)$ it is easy to see that $h^{1}\left(Y, \mathcal{E} \otimes \mathcal{O}_{Y}\left(-z \gamma-\rho^{*} P\right)\right)=$ 0 .

Let us consider any effective divisor $D \equiv z \pi^{*} \gamma$ on $X$ and the exact sequence: $0 \rightarrow T-D \rightarrow T \rightarrow T \otimes \mathcal{O}_{D} \rightarrow 0$. The natural map $|T| \rightarrow\left|T_{\mid D}\right|$ is surjective as $h^{1}(X, T-D)=h^{1}\left(Y, \mathcal{E} \otimes \mathcal{O}_{Y}(-z \gamma)\right)=0$ by the above vanishing. A similar argument shows that if $D \equiv z \pi^{*} \gamma+\pi^{*} \rho^{*} P$, the natural map is surjective too, for any point $P \in C$.

Now we proceed as in the proof of Theorem 4.2 after recalling that, in our assumptions, on $Y$ there are no $\left|C_{0}+\pi^{*} \varkappa\right|$-lines except for the ruling by Lemma 2.1 .

Now we can repeat the proof of Theorem 4.2, more or less verbatim. We have to use Lemma 5.1 with $r=\left(C_{0}+\pi^{*} \varkappa\right)^{2}=(2 x-e)\left(\right.$ or $\left.r=\left(C_{0}+\pi^{*} \varkappa\right)^{2}+1=(2 x-e)+1\right)$ instead of Lemma 4.1. Note that, in any case, assumption 0$)$ implies that $r \geq 2$. The only difference is that here $h^{0}\left(\Gamma_{i}, T_{\mid \Gamma_{i}}\right)=h^{0}\left(\Gamma_{2}, T_{\mid \Gamma_{2}}\right)=\left(a_{l}+a_{m}\right)(x-e)+b_{l}+b_{m}$ for any $i \geq 2$, so that the last condition has to be: $\left(a_{l}+a_{m}\right)(x-e)+b_{l}+b_{m} \geq 2(z-1) r+2$ in all cases, i.e. we need assumption 6).

\section{VERY AMPLENESS THROUGH GENERAL POSITION}

In this section we consider some very special rank 2 vector bundles over $Y=\mathbf{F}_{1}$. The aim of Section 6 will be evident in Section 7 .

Definition 6.1. Let $W^{\prime}$ be a 0 -dimensional scheme of lenght $w^{\prime}$ in $\mathbb{P}^{2}$ consisting of $w^{\prime}$ simple points. These points are said to be in general position on $\mathbb{P}^{2}$ if, for any positive integer $k, h^{0}\left(\mathbb{P}^{2}, \mathcal{I}_{W^{\prime}}(k)\right)=\max \left\{h^{0}\left(\mathbb{P}^{2}, \mathcal{O}_{\mathbb{P}^{2}}(k)\right)-w^{\prime}, 0\right\}=\max \left\{\left(\begin{array}{c}k+2 \\ 2\end{array}\right)-\right.$ $\left.w^{\prime}, 0\right\}$. Let $s: Y \rightarrow \mathbb{P}^{2}$ be the blow up of $\mathbb{P}^{2}$ at one point $P_{0}$, and let $W \subset Y$ be a 0-dimensional scheme consisting of $w$ simple points none of which lie on the exceptional divisor. These points are said to be in general position on $Y$ if $P_{0} \cup s(W)$ consists of $w^{\prime}=w+1$ distinct points in general position on $\mathbb{P}^{2}$.

Definition 6.1 can be reinterpreted in terms of cohomological vanishing.

Lemma 6.1. Let $s: Y \rightarrow \mathbb{P}^{2}$ be the blow up of $\mathbb{P}^{2}$ at one point $P_{0}$. Let $W$ be a set of $w$ distinct points on $Y$ in general position according to definition 6.1 ; let $D \equiv$ $a C_{0}+b f$ be a divisor on $Y$ such that $a \geq 0, b \geq 1$ and $b \geq a$. Let us assume that $h^{0}(Y, D) \geq w$, then $h^{1}\left(Y, D \otimes \mathcal{I}_{W}\right)=0$.

Proof. Let us consider the exact sequence: $0 \rightarrow D \otimes \mathcal{I}_{W} \rightarrow D \rightarrow D \otimes \mathcal{O}_{W} \rightarrow 0$. 
As $H^{1}(Y, D)=0$ we get our vanishing if (ad only if) the restriction map $r$ : $H^{0}(Y, D) \rightarrow H^{0}\left(W, D \otimes \mathcal{O}_{W}\right)$ is surjective. Let $l$ be the pull back of the generator of $\operatorname{Pic}\left(\mathbb{P}^{2}\right)$ and let $l_{0}$ be the exceptional divisor for $s$, then $D=b l-(b-a) l_{0}$ and, by assumptions, $D$ is an effective divisor. Hence $r$ is surjective if and only if the 0 dimensional scheme $s(W)$ imposes independent conditions on plane curves of degree $b$, having a point of multiplicity at least $b-a$ at $P_{0}$. This condition, using the same language, definitions and notation introduced in $[\mathrm{C}-\mathrm{M}]$, pag. 192, is equivalent to the quasi-homogeneous linear system $\mathcal{L}(b, b-a, w, 1)$ not being special. This in turn follows from Theorem 8.1 and Lemma 7.1 of $[\mathrm{C}-\mathrm{M}]$ as $P_{0} \cup s(W)$ are in general position in $\mathbb{P}^{2}$.

Now, let $L \equiv C_{0}+(5-h) f$ and $M \equiv 2 C_{0}+h f$ be two line bundles on $Y$ with $h \geq 3$. Let us fix an integer $y$ such that $-2 \leq y \leq 4$ and let us choose a set $W$ of $w=h+y \geq 1$ distinct points on $Y$ in general position according to definition 6.1 ; in particular each fibre contains at most one point of $W$. On our surface $K_{Y} \equiv-2 C_{0}-3 f$ so that $\left|K_{Y}+M-L\right|=\left|-C_{0}+(2 h-8) f\right|=\emptyset$ and therefore we can apply Griffiths-Harris theorem on the existence of rank 2 vector bundles on surfaces (see [D-L], Theorem 3.13 and its proof) and we get an exact sequence as the following: $0 \rightarrow \mathcal{O}_{Y} \rightarrow \mathcal{E}_{y}^{\prime} \rightarrow(M-L) \otimes \mathcal{I}_{W} \rightarrow 0$; by tensorizing it with $L$ we get rank 2 vector bundles $\mathcal{E}_{y}$ :

$$
0 \rightarrow L \rightarrow \mathcal{E}_{y} \rightarrow M \otimes \mathcal{I}_{W} \rightarrow 0
$$

such that $c_{1}\left(\mathcal{E}_{y}\right) \equiv 3 C_{0}+5 f, c_{2}\left(\mathcal{E}_{y}\right)=8+y$. In this section we prove very amplenes results for $\mathcal{E}_{y}$, with $-2 \leq y \leq 3$, for suitable sets $W$ of $h+y$ generic points of $Y$. These results are presented in Theorems 6.6 and 6.9 after a number of preparatory Lemmas.

Firstly we have the following.

Proposition 6.2. Let $\mathcal{E}_{y}$ be any vector bundle defined as above by $(*)$. Let $\bar{f}$ be any fixed fibre of $Y$. Let $\gamma$ be a smooth element of $\left|C_{0}+f\right|$ passing through at most two points of $W$. Then:

i) the restriction $\mathcal{E}_{y \mid \bar{f}}$ is isomorphic to $\mathcal{O}_{\mathbb{P}^{1}}(1) \oplus \mathcal{O}_{\mathbb{P}^{1}}(2)$, hence very ample;

ii) if $h=3$ then $h^{1}\left(Y, \mathcal{E}_{y} \otimes \mathcal{O}_{Y}(-\bar{f})\right)=0$ for $-2 \leq y \leq 3$;

iii) if $h=4$ then $h^{1}\left(Y, \mathcal{E}_{y} \otimes \mathcal{O}_{Y}(-\bar{f})\right)=0$ for $-2 \leq y \leq 4$;

iv) if $h=3$ the restriction $\mathcal{E}_{y \mid C_{0}}$ is very ample;

$v)$ if $h=4$ the restriction $\mathcal{E}_{y \mid C_{0}}$ is very ample or, possibly, the rational map associated to the linear system of the tautological divisor in $\mathbb{P}\left(\mathcal{E}_{y \mid C_{0}}\right)$ is a birational morphism, contracting only $C_{0}$ at a singular double point;

vi) if $h=3$ or $h=4, h^{1}\left(Y, \mathcal{E}_{y} \otimes \mathcal{O}_{Y}\left(-C_{0}\right)\right)=0$;

vii) if $h=3$ or $h=4$, the restriction $\mathcal{E}_{y \mid \gamma}$ is very ample;

viii) if $h=3$ then $h^{1}\left(Y, \mathcal{E}_{y} \otimes \mathcal{O}_{Y}(-\gamma)\right)=0$ for $-2 \leq y \leq 2$

ix) if $h=4$ then $h^{1}\left(Y, \mathcal{E}_{y} \otimes \mathcal{O}_{Y}(-\gamma)\right)=0$ for $-2 \leq y \leq 3$.

Proof. For $i$ ) we restrict $(*)$ to $\bar{f}$ and we get the exact sequence:

$0 \rightarrow \mathcal{O}_{\mathbb{P}^{1}}(1+\varepsilon) \rightarrow \mathcal{E}_{y \mid \bar{f}} \rightarrow \mathcal{O}_{\mathbb{P}^{1}}(2-\varepsilon) \rightarrow 0$

where $\varepsilon=1$ or $\varepsilon=0$ according to whether $\bar{f}$ contains one point of $W$ or not. Note that, in any case, $\mathcal{E}_{y \mid \bar{f}} \simeq \mathcal{O}_{\mathbb{P}^{1}}(1) \oplus \mathcal{O}_{\mathbb{P}^{1}}(2)$, hence very ample.

For $i$ ) and $i i i)$ we tensorize $(*)$ by $\mathcal{O}_{Y}(-\bar{f})$ and we get: $0 \rightarrow C_{0}+(4-h) f \rightarrow \mathcal{E}_{y} \otimes \mathcal{O}_{Y}(-\bar{f}) \rightarrow\left(2 C_{0}+h f-\bar{f}\right) \otimes \mathcal{I}_{W} \rightarrow 0$.

In any case $h^{1}\left(Y, C_{0}+(4-h) f\right)=0$. Moreover $h^{1}\left(Y,\left(2 C_{0}+h f-\bar{f}\right) \otimes \mathcal{I}_{W}\right)=0$, 
by Lemma 6.1 , if $h^{0}\left(Y, 2 C_{0}+(h-1) f\right)=3 h-3 \geq w=h+y$.

Hence $h^{1}\left(Y, \mathcal{E}_{y} \otimes \mathcal{O}_{Y}(-\bar{f})\right)=0$ for $y \leq 3$ when $h=3$ and for $y \leq 4$ when $h=4$.

For $i v$ ) and $v$ ) we restrict $(*)$ to $C_{0}$ and we get the exact sequence (recall that $\left.W \cap C_{0}=\emptyset\right): 0 \rightarrow \mathcal{O}_{\mathbb{P}^{1}}(4-h) \rightarrow \mathcal{E}_{y \mid C_{0}} \rightarrow \mathcal{O}_{\mathbb{P}^{1}}(h-2) \rightarrow 0$. If $h=3$ then $\mathcal{E}_{y \mid C_{0}}$ is very ample. If $h=4$, we have two cases: $\mathcal{E}_{y \mid C_{0}}=\mathcal{O}_{\mathbb{P}^{1}}(1) \oplus \mathcal{O}_{\mathbb{P}^{1}}(1)$, very ample, or $\mathcal{E}_{y \mid C_{0}}=\mathcal{O}_{\mathbb{P}^{1}} \oplus \mathcal{O}_{\mathbb{P}^{1}}(2)$; in this second case it is well known that $\mathbb{P}\left(\mathcal{E}_{y \mid C_{0}}\right) \simeq \mathbf{F}_{2}$ and the tautological linear system gives rise to a birational morphism sending $\mathbf{F}_{2}$ into a quadric cone of rank 3 , singular only at its vertex, where the curve $C_{0}$ is contracted.

For $v i)$ we tensorize $(*)$ by $\mathcal{O}_{Y}\left(-C_{0}\right)$ and we get the exact sequence (recall that $\left.W \cap C_{0}=\emptyset\right): 0 \rightarrow(5-h) f \rightarrow \mathcal{E}_{y} \otimes \mathcal{O}_{Y}\left(-C_{0}\right) \rightarrow\left(C_{0}+h f\right) \otimes \mathcal{I}_{W} \rightarrow 0$; now $h^{1}(Y,(5-h) f)=0$ and $h^{1}\left(Y,\left(C_{0}+h f\right) \otimes \mathcal{I}_{W}\right)=0$ by Lemma 6.1 as $h^{0}\left(Y, C_{0}+h f\right)=$ $2 h+1 \geq w=h+y$.

For vii) we restrict $(*)$ to $\gamma$ and we get the exact sequence: $0 \rightarrow \mathcal{O}_{\mathbb{P}^{1}}(5-h+\varepsilon) \rightarrow \mathcal{E}_{y \mid \gamma} \rightarrow \mathcal{O}_{\mathbb{P}^{1}}(h-\varepsilon) \rightarrow 0$ where $\varepsilon=0,1,2$ according to whether $\gamma$ passes through $0,1,2$ points of $W$. In any case $\mathcal{E}_{y \mid \gamma}$ is very ample.

For viii) and $i x)$ we tensorize $(*)$ by $\mathcal{O}_{Y}(-\gamma)$ and we get the exact sequence: $0 \rightarrow(4-h) f \rightarrow \mathcal{E}_{y} \otimes \mathcal{O}_{Y}(-\gamma) \rightarrow\left(2 C_{0}+h f-\gamma\right) \otimes \mathcal{I}_{W} \rightarrow 0$.

In any case $h^{1}(Y,(4-h) f)=0$. Moreover $h^{1}\left(Y,\left(2 C_{0}+h f-\gamma\right) \otimes \mathcal{I}_{W}\right)=0$, by Lemma 6.1 , if $h^{0}\left(Y, 2 C_{0}+h f-\gamma\right)=2 h-1 \geq w=h+y$. Hence $h^{1}\left(Y, \mathcal{E}_{y} \otimes \mathcal{O}_{Y}(-\gamma)\right)=0$ for $y \leq 2$ when $h=3$ and for $y \leq 3$ when $h=4$.

Now we prove the following Lemmas.

Lemma 6.3. Let $\mathcal{E}_{y}$ be any vector bundle defined as above by $(*)$. Let $A \equiv C_{0}+x f$ be a divisor on $Y$. Then $\mathcal{E}_{y} \otimes \mathcal{O}_{Y}(A)$ is very ample for $x>0$.

Proof. Let us tensorize $(*)$ by $\mathcal{O}_{Y}(A)$. We get:

$$
0 \rightarrow L+A \rightarrow \mathcal{E}_{y} \otimes \mathcal{O}_{Y}(A) \rightarrow(M+A) \otimes \mathcal{I}_{W} \rightarrow 0 .
$$

By Proposition 4.2 of [B-D-S] it suffices to show that $\mathcal{E}_{y} \otimes \mathcal{O}_{Y}(A)$ is $1-$ jet ample and, by Proposition 4.1 of [B-D-S] it suffices to show that $\mathcal{E}_{y} \otimes \mathcal{O}_{Y}(A-B)$ is generated by global sections where $B$ is a very ample divisor such that $B \equiv C_{0}+2 f$. In other words, it suffices to show that $\mathcal{E}_{y} \otimes \mathcal{O}_{Y}(x f)$ is generated by global sections for $x>>0$. Let us consider $\mathbb{P}\left(\mathcal{E}_{y} \otimes \mathcal{O}_{Y}(x f)\right)$ and let us consider its tautological divisor $\mathcal{T}$, we have only to show that $|\mathcal{T}|$ has no base points.

Let us tensorize $(*)$ by $\mathcal{O}_{Y}(x f)$. We get:

$$
0 \rightarrow L+x f \rightarrow \mathcal{E}_{y} \otimes \mathcal{O}_{Y}(x f) \rightarrow(M+x f) \otimes \mathcal{I}_{W} \rightarrow 0 .
$$

Let us fix any fibre $\bar{f}$ of $Y$. By arguing as in the proof of Proposition $6.2 i$ ), we have that $\left[\mathcal{E}_{y} \otimes \mathcal{O}_{Y}(x f)\right]_{\mid \bar{f}}$ is very ample for $x>>0$. By arguing as in the proof of Proposition $6.2 i i)$ and $i i i)$, we have that $h^{1}\left(Y, \mathcal{E}_{y} \otimes \mathcal{O}_{Y}(x f-\bar{f})\right)=0$ for $x>>0$. Hence, we get that $\mathcal{T}_{\mid \bar{f}}$ is very ample and $|\mathcal{T}| \rightarrow\left|\mathcal{T}_{\mid \bar{f}}\right|$ is surjective. Now, by contradiction, let us assume that $|\mathcal{T}|$ has a base point $P$ and let $\bar{f}$ be the unique fibre of $Y$ passing through $\pi(P) ; P$ would be a base point also for $\left|\mathcal{T}_{\mid \bar{f}}\right|$, but this is not possible as $\mathcal{T}_{\mid \bar{f}}$ is very ample.

Lemma 6.4. Let $\mathcal{E}_{y}$ be any vector bundle defined as above by $(*)$ and let $\bar{f}$ be any fixed fibre of $Y$. Let $S_{1}$ be the rational ruled surface $\mathbb{P}\left(\mathcal{E}_{y \mid \bar{f}}\right)$ and let $\Gamma_{0}$ and $\varphi$ be 
the standard generators of $N u m\left(S_{1}\right) \simeq \operatorname{Pic}\left(S_{1}\right)$. Let $T$ be the tautological divisor of $X:=\mathbb{P}\left(\mathcal{E}_{y}\right)$ as usual and let $A \equiv C_{0}+x f$ be a divisor on $Y$ with $x>>0$. We have the following:

i) $S_{1} \simeq \mathbf{F}_{1}, T_{\mid S_{1}} \equiv \Gamma_{0}+2 \varphi$ is very ample and for any generic smooth element $S_{2} \in\left|T+\pi^{*} A\right|$ the intersection $S_{2} \cap S_{1}$ is a smooth rational irreducible curve $C \equiv \Gamma_{0}+3 \varphi$, moreover $\left|T_{\mid S_{1}}\right|_{\mid C} \simeq\left|T_{\mid S_{1}}\right|$;

ii) let $\xi \in X^{[2]}$ be any subscheme contained in a smooth surface as $S_{1}$ (i.e. $\left.\xi \in S_{1}^{[2]}\right)$, then $\xi$ is separated by $|T|$ for $-2 \leq y \leq 3$ if $h=3$ and for $-2 \leq y \leq 4$ if $h=4$, moreover $|T|$ has no base points;

iii) the generic surface $S_{2} \in\left|T+\pi^{*} A\right|$ is isomorphic to the the blow up of $Y$ at $5 x+9+y$ distinct points, hence to the blow up of $\mathbb{P}^{2}$ at $5 x+10+y$ distinct points; if we generate $\operatorname{Num}\left(S_{2}\right)$ with the pull back l of the generator of $\operatorname{Pic}\left(\mathbb{P}^{2}\right)$, the pull back $l_{0}$ of $C_{0} \in Y$ and the classes of the exceptional divisors, we have that $T_{\mid S_{2}} \equiv(x+5) l-(x+1) l_{0}-l_{1} \cdots-l_{5 x+9+y},|T| \simeq\left|T_{\mid S_{2}}\right|$ and $h^{0}\left(S_{2}, T_{\mid S_{2}}\right) \geq 11-y$.

Proof. $i$ ) The first conclusions follow from Proposition 6.2, part $i$ ). Moreover we have that $S_{2 \mid S_{1}}=\left(T+\pi^{*} A\right)_{\mid S_{1}} \equiv \Gamma_{0}+2 \varphi+\varphi$ and for generic $S_{2} \in\left|T+\pi^{*} A\right|$ the intersection $C:=S_{2} \cap S_{1}$ is a smooth irreducible curve because $T+\pi^{*} A$ is the tautological divisor of $\mathbb{P}\left(\mathcal{E}_{y} \otimes \mathcal{O}_{Y}(A)\right)$, hence it is a very ample divisor of $X$ by Lemma 6.3. $C$ is rational being a section of $\mathbf{F}_{1}$.

Let us consider the exact sequence $0 \rightarrow T_{\mid S_{1}}-C \rightarrow T_{\mid S_{1}} \rightarrow\left(T_{\mid S_{1}}\right)_{\mid C} \rightarrow 0$ on $S_{1}$, we have $h^{i}\left(S_{1}, T_{\mid S_{1}}-C\right)=h^{i}\left(S_{1}, \Gamma_{0}+2 \varphi-\left(\Gamma_{0}+3 \varphi\right)\right)=h^{i}\left(S_{1},-\varphi\right)=0$ for $i=0,1$, so that $\left|T_{\left|S_{1}\right|}\right|_{\mid C} \simeq \mid T_{\left|S_{1}\right|}$, note that $h^{0}\left(S_{1}, T_{\mid S_{1}}\right)=5$.

ii) Let us look at the exact sequence: $0 \rightarrow T-S_{1} \rightarrow T \rightarrow T_{\mid S_{1}} \rightarrow 0$ on $X$. We know that $T_{\mid S_{1}}$ is very ample in any case, so that $\left|T_{\mid S_{1}}\right|$ separates $\xi$, moreover we have $h^{1}\left(X, T-S_{1}\right)=0$ for $-2 \leq y \leq 3$, if $h=3$, by Proposition 6.2, part ii), and $h^{1}\left(X, T-S_{1}\right)=0$ for $-2 \leq y \leq 4$, if $h=4$, by Proposition 6.2 , part iii). Hence the map $|T| \rightarrow\left|T_{\left|S_{1}\right|}\right|$ is surjective and we can separate $\xi$ by $|T|$.

Now, let us assume by contradiction that $|T|$ has a base point $P$ and let $f_{P}$ be the fibre of $Y$ passing through $\pi(P)$. As we have seen the map $|T| \rightarrow\left|T_{\mid S_{1}}\right|$ is surjective, where $S_{1}=\mathbb{P}\left(\mathcal{E}_{y \mid f_{P}}\right)$, then $P$ would be a base point for $\left|T_{\mid S_{1}}\right|$ too. But it is not possible because $T_{\mid S_{1}}$ is very ample.

iii) Recall that, for $x>0, \mathcal{E}_{y} \otimes \mathcal{O}_{Y}(A)$ is very ample by Lemma 6.3. It is well known that $c_{2}\left[\mathcal{E}_{y} \otimes \mathcal{O}_{Y}(A)\right]$ is the zero cycle of the zero locus of a generic section of $\mathcal{E}_{y} \otimes \mathcal{O}_{Y}(A)$ and that a smooth element $S_{2} \in\left|T+\pi^{*} A\right|$ is isomorphic to the blow up of $Y$ exactly at the $\operatorname{deg}\left\{c_{2}\left[\mathcal{E}_{y} \otimes \mathcal{O}_{Y}(A)\right]\right\}=5 x+9+y$ points which are the zero locus of the corresponding section of $\mathcal{E}_{y} \otimes \mathcal{O}_{Y}(A)$ (see [B-S] Theorem 11.1.2.). Moreover $H^{1}(Y, L+A)=0$ for $x>>0$, hence $H^{0}\left(Y, \mathcal{E}_{y} \otimes \mathcal{O}_{Y}(A)\right)=$ $H^{0}(Y, L+A) \oplus H^{0}\left(Y,(M+A) \otimes \mathcal{I}_{W}\right)$ and the zero locus of any section $\sigma=\sigma^{\prime}+\sigma^{\prime \prime}$ of $\mathcal{E}_{y} \otimes \mathcal{O}_{Y}(A)$ is a group of $5 x+9+y$ points belonging to $\left(\sigma^{\prime \prime}\right)_{0}$ (note that this is independent of $h)$. For generic $\sigma$ the zero locus $\left(\sigma^{\prime \prime}\right)_{0}$ is a smooth curve and $(\sigma)_{0}$ is a set of points linearly equivalent, on this curve, to the intersection with $\left(\sigma^{\prime}\right)_{0}$, so that we can assume that they are all distinct.

Let $C_{0}^{\prime}, f^{\prime}, l_{1}, \ldots . ., l_{5 x+9+y}$ be the generators of $\operatorname{Num}\left(S_{2}\right)$ (the classes of the pull back of $C_{0}, f$ and the $5 x+9+y$ exceptional divisors of the blow up). The Wu-Chern relation for $\mathcal{E}_{y} \otimes \mathcal{O}_{Y}(A)$ (see [G-H] pag. 606) implies that $\left(T+\pi^{*} A\right)^{2}=\pi^{*}\left\{c_{1}\left[\mathcal{E}_{y} \otimes\right.\right.$ $\left.\left.\mathcal{O}_{Y}(A)\right]\right\}\left(T+\pi^{*} A\right)-c_{2}\left[\mathcal{E}_{y} \otimes \mathcal{O}_{Y}(A)\right]$. Hence $\left(T+\pi^{*} A\right)_{\mid S_{2}} \equiv\left(\pi_{\mid S_{2}}\right)^{*}\left\{c_{1}\left[\mathcal{E}_{y} \otimes\right.\right.$ $\left.\left.\mathcal{O}_{Y}(A)\right]\right\}-l_{1} \ldots-l_{5 x+9+y} \equiv\left(\pi_{\mid S_{2}}\right)^{*}\left(3 C_{0}+5 f+2 A\right)-l_{1} \ldots-l_{5 x+9+y}$ and $T_{\mid S_{2}} \equiv$ $\left(\pi_{\mid S_{2}}\right) *\left(3 C_{0}+5 f+A\right)-l_{1} \ldots-l_{5 x+9+y} \equiv 4 C_{0}^{\prime}+(5+x) f^{\prime}-l_{1} \ldots-l_{5 x+9+y}$. 
As $Y$ is the blow up of $\mathbb{P}^{2}$ at one point $P_{0}, S_{2}$ is the blow up of $\mathbb{P}^{2}$ at $5 x+10+y$ points, so we can also generate $\operatorname{Num}\left(S_{2}\right)$ with the pull back $l$ of the numerical class of a line in $\mathbb{P}^{2}$ and the classes of the $5 x+10+y$ exceptional divisors. If $l_{0}$ is the class of the pull back of the exceptional divisor of the blow up of $\mathbb{P}^{2}$ at $P_{0}$ we have $C_{0}^{\prime} \equiv l_{0}$ and $f^{\prime} \equiv l-l_{0}$ so that: $T_{\mid S_{2}} \equiv(x+5) l-(x+1) l_{0}-l_{1} \ldots-l_{5 x+9+y}$. It is easy to see that $h^{0}\left(S_{2}, T_{\mid S_{2}}\right) \geq 11-y$. Note that $h^{0}\left(S_{2}, T_{\mid S_{2}}\right)=11-y$ if the $5 x+10+y$ points are in general position, but this fact is not known a priori.

Now let us consider the exact sequence: $0 \rightarrow T-S_{2} \rightarrow T \rightarrow T_{\mid S_{2}} \rightarrow 0$ on $X$. As $T-S_{2}=-\pi^{*} A$ we have that $h^{0}\left(X,-\pi^{*} A\right)=0$ and $h^{1}\left(X,-\pi^{*} A\right)=h^{1}(Y,-A)=0$, so that $H^{0}(X, T)=H^{0}\left(S_{2}, T_{\mid S_{2}}\right)$ hence $|T| \simeq\left|T_{\mid S_{2}}\right|$.

Lemma 6.5. Let $\mathcal{E}_{y}$ be any vector bundle defined as above by $(*)$. Let $\xi \in X^{[2]}$ be any subscheme of $X$ having its support at a single point $P \in X$. Then $|T|$ separates $\xi$ for $-2 \leq y \leq 3$ if $h=3$ and for $-2 \leq y \leq 4$ if $h=4$.

Proof. We will show that assumptions $\beta$ ) and $\gamma$ ) of Proposition 3.5 are satisfied with $A \equiv C_{0}+x f, x>>0$, and $B \equiv f$. It is easy to see that $\gamma$ ) it is true for $x>>0$, so we have to prove that $\beta$ ) is true for any point $P$ and any direction $\underline{q \in} \mathbb{T}_{P}(X)$. In other words, we have to show that there exists a smooth section $\tau \in$ $\overline{|T|}$ such that $(\tau)_{0}$ passes through $P$ and its tangent space at $P$ does not contain $\underline{q}$.

Let us consider $\pi(P)$ and the fibre $f_{P}$ of $Y$ passing through $\pi(P)$. Let us choose a smooth surface $S_{2} \in\left|T+\pi^{*} A\right|$ passing through $P$ (recall that $T+\pi^{*} A$ is very ample for $x>>0$ ) and a smooth $S_{1}:=\pi^{-1}\left(f_{P}\right)$; so $\left.\left.\beta\right) i\right)$ is fullfilled.

By Lemma $6.4 \mathrm{ii}$ ) we can assume that $\underline{q} \notin \mathbb{T}_{P}\left(S_{1}\right)$.

By recalling that $T_{\mid S_{1}}$ is very ample in any case we can choose $\sigma_{1} \in\left|T_{\mid S_{1}}\right|$ such that $\left(\sigma_{1}\right)_{0}$ is a smooth curve, passing through $P$, with tangent vector $\underline{t} \in \mathbb{T}_{P}\left(S_{1}\right)$ and $\beta$ ) $i i)$ is fulfilled. Obviously $\underline{t} \neq q$. We can also choose a generic smooth element $S_{2} \in\left|T+\pi^{*} A\right|$ such that $S_{2}$ cuts transversely $S_{1}$ along a smooth rational curve $C$, (hence $\left.\mathbb{T}_{P}\left(S_{1}\right) \cap \mathbb{T}_{P}\left(S_{2}\right)=\mathbb{T}_{P}(C)\right)$ and $S_{2}$ cuts transversely $\left(\sigma_{1}\right)_{0}$ at 4 distinct points $P=R_{1}, \ldots, R_{4} \in C$ by Lemma $\left.6.4 i\right)$. The independent vectors $\underline{t}$ and $\underline{q} \in \mathbb{T}_{P}(X)$ generates a 2 -plane in $\mathbb{T}_{P}(X)$ cutting $\mathbb{T}_{P}\left(S_{2}\right)$ along a vector $\underline{w}$. Now we choose a vector $\underline{v} \in \mathbb{T}_{P}\left(S_{2}\right), \underline{v} \neq \underline{w}$, and we consider the linear subsystem $\Lambda$ of $\left|T_{\mid S_{2}}\right|=\left|(x+5) L-(x+1) l_{0}-l_{1} \ldots-l_{5 x+9+y}\right|$ (see Lemma $6.4 \mathrm{iii}$ )) given by those elements passing through $P=R_{1}, \ldots, R_{4}$ and such that their zero-loci are tangent to $\underline{v}$ at $P$. This is possible because $h^{0}(X, T)=h^{0}\left(S_{2}, T_{\mid S_{2}}\right) \geq 11-y$, (see Lemma $6.4 \mathrm{iii})$ ), and therefore the (projective) dimension of $\Lambda$ is $\geq 5-y \geq 1$.

Now we want to show that not all the zero loci of the elements of $\Lambda$ are singular at $P$. In fact any element of $\Lambda$ whose zero-locus is singular at $P$ comes from a degree $x+5$ plane curve $\bar{C}$ intersecting a line $\bar{l}$ passing through $P_{0}$ (corresponding to $C$ ) with multiplicity at least $x+6$. Hence $\bar{l}$ is an irreducuble component of $\bar{C}$ and the zero-locus of the corresponding element of $\Lambda$ contains $C$. Therefore the zero-loci of all elements of $\Lambda$ are singular at $P$ if and only if $\Lambda=\left|T_{\mid S_{2}}-C\right|$. But this is a fixed subspace of $\left|T_{\mid S_{2}}\right|$, whose sections have zero-loci having at most a finite number of fixed tangent vectors at $P$. If $\Lambda=\left|T_{\mid S_{2}}-C\right|$ all zero-loci of $\Lambda$ are reducible as the union of $C$ and other curves passing through $P$ with the same tangent vector $\underline{v}$, so that it suffices to change suitably the choice of $\underline{v}$ to avoid this case.

In conclusion we can assume that not all zero-loci of the elements of $\Lambda$ are singular at $P$, hence that not all of them contain $C$. Hence there exists a section 
$\sigma_{2} \in \Lambda \subseteq\left|T_{\mid S_{2}}\right|$ whose zero-locus is smooth at $P$, having $\underline{v}$ as tangent vector, such

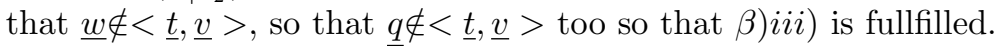

As $H^{0}(X, T)=H^{0}\left(S_{2}, T_{\mid S_{2}}\right)$ we get that there exists $\tau \in|T|$ such that $\tau_{\mid S_{2}}=\sigma_{2}$ and $(\tau)_{0}$ does not contain $S_{1}$ because $\left(\sigma_{2}\right)_{0}$ does not contain $C$. Hence $\sigma_{1}^{\prime}:=\tau_{\mid S_{1}}$ is a non zero section of $\left|T_{\mid S_{1}}\right|$ and we have $\sigma_{1}^{\prime} \in<\sigma_{1}>$ because $\sigma_{1}^{\prime}$ and $\sigma_{1}$ cut the same divisor $R_{1}+R_{2}+R_{3}+R_{4}$ on $C$ and $\left|T_{\left|S_{1}\right|}\right|_{\mid C} \simeq\left|T_{\mid S_{1}}\right|$ by Lemma $\left.6.4 i\right)$. By choosing suitably the generator of $\left\langle\sigma_{1}\right\rangle$ we can assume that $\tau_{\mid S_{1}}=\sigma_{1}$ so that $\beta) i v$ ) is fulfilled a fortiori by choosing $\sigma:=\tau_{\mid S_{1} \cup S_{2}}$.

Now we can prove the following result, stating the very ampleness of almost all vector bundles $\mathcal{E}_{y}$ when $h=3$.

Theorem 6.6. Let $\mathcal{E}_{y}$ be any vector bundle defined as above by $(*)$ with $-2 \leq y \leq 2$ and $h=3$. Then $\mathcal{E}_{y}$ is very ample.

Proof. Let $T$ be the tautological divisor of $X=\mathbb{P}\left(\mathcal{E}_{y}\right)$, let $\xi$ be any fixed element of $X^{[2]}$ and let $s: Y \rightarrow \mathbb{P}^{2}$ be the blow up of $\mathbb{P}^{2}$ at $P_{0}$. We have to prove that $|T|$ separates $\xi$. By Lemmas 6.5 and 6.4 we know that we have to consider only the cases in which the support of $\xi$ consists of a couple of distinct points $P, Q$ projecting on different fibres of $Y$.

Case 1: neither $\pi(P)$ nor $\pi(Q)$ belong to $C_{0}$. We use a slightly different version of the proof of Theorem 4.2 i.e. we use the linear system $\left|C_{0}+f\right|$ which is not very ample. However, in this case, there exists a smooth element $\gamma \in\left|C_{0}+f\right|$ passing through $\pi(P)$ and $\pi(Q)$. Moreover $\gamma$ passes through two points of $W$ at most, because $\gamma$ corresponds to the unique line passing through $s[\pi(P)]$ and $s[\pi(Q)]$ on $\mathbb{P}^{2}$ and $s(W)$ is a set of points in general position on $\mathbb{P}^{2}$. Very ampleness of $\mathcal{E}_{y}$ follows from Proposition 6.2 vii) and viii) as $T_{\mid \mathbb{P}\left(\mathcal{E}_{y \mid \gamma}\right)}$ is very ample and $|T| \rightarrow \mid T_{\left|\mathbb{P}\left(\mathcal{E}_{y \mid \gamma}\right)\right|}$ is surjective.

Case 2: $\pi(P)$ and $\pi(Q)$ belong to $C_{0}$. Very ampleness of $\mathcal{E}_{y}$ follows from Proposition $6.2 i v)$ and $v i)$ as $T_{\mid \mathbb{P}\left(\mathcal{E}_{y \mid C_{0}}\right)}$ is very ample and $|T| \rightarrow\left|T_{\mid \mathbb{P}\left(\mathcal{E}_{y \mid C_{0}}\right)}\right|$ is surjective.

Case 3: $\pi(Q) \in C_{0}$ and $\pi(P) \notin C_{0}$. Let $f_{P}$ be the fibre of $Y$ passing through $\pi(P)$ and let $\Sigma \subset X$ be the reducible surface $\mathbb{P}\left(\mathcal{E}_{y \mid C_{0}}\right) \cup \mathbb{P}\left(\mathcal{E}_{y \mid f_{P}}\right)$. Let $R \in Y$ be the unique point $f_{P} \cap C_{0}$ so that $\mathbb{P}\left(\mathcal{E}_{y \mid C_{0}}\right) \cap \mathbb{P}\left(\mathcal{E}_{y \mid f_{P}}\right)=\mathrm{F}_{R}$ (as in the proof of Proposition 3.5). By Proposition $6.2 \mathrm{iv})$, we know that $T_{\mid \mathbb{P}\left(\mathcal{E}_{y \mid C_{0}}\right)}$ is very ample, so that we can take a smooth element $\sigma_{2} \in\left|T_{\mid \mathbb{P}\left(\mathcal{E}_{y \mid C_{0}}\right)}\right|$ such that $Q \notin\left(\sigma_{2}\right)_{0}$ and $\left(\sigma_{2}\right)_{0}$ cuts $\mathrm{F}_{R}$ transversely at a point $H$. By Proposition $\left.6.2 i\right)$, and $\left.i i\right)$, we know

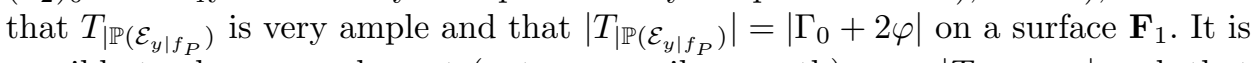
possible to choose an element (not necessarily smooth) $\sigma_{1} \in\left|T_{\mid \mathbb{P}\left(\mathcal{E}_{y \mid f_{P}}\right)}\right|$ such that $\left(\sigma_{1}\right)_{0}$ passes through $P$ and cuts $\mathrm{F}_{R}$ transversely at $H$. Note that $\mathbf{F}_{1}$ is embedded by $\left|\Gamma_{0}+2 \varphi\right|$ as a scroll in $\mathbb{P}^{4}$, in such a way that $\mathrm{F}_{R}$ is a fibre of the scroll, but $P \notin \mathrm{F}_{R}$, hence it is not possible that all hyperplanes passing through $P$ and $H$ contain the line $\mathrm{F}_{R}$ in $\mathbb{P}^{4}$.

Now the pair $\left(\sigma_{1}, \sigma_{2}\right)$ is a section of $\left|T_{\mid \Sigma}\right|$ separating $P$ from $Q$ and, by Proposition 6.2 viii), we can lift this element to an element of $|T|$ acting in the same way and we are done. In fact, note that the proof of Proposition 6.2 viii) works even when $\gamma=C_{0} \cup f_{P}$.

Obviously if $\pi(Q) \notin C_{0}$ and $\pi(P) \in C_{0}$ we can interchange the roles of $P$ and $Q$ in the previous argument. 
We can also prove the very ampleness of $\mathcal{E}_{3}$ when $h=4$, but we need other Lemmas.

Lemma 6.7. Let $P_{0}, P_{1}, \ldots, P_{8}$ be 9 distinct points in $\mathbb{P}^{2}$, lying on a smooth cubic curve $\mathcal{C}$. Assume that the complete linear system $\mathcal{L}$ of quartics passing through them has no base points. Then $\mathcal{L}$ is very ample.

Proof. Let $S$ be the blow up of $\mathbb{P}^{2}$ at $P_{0}, P_{1}, \ldots, P_{8}$. We can generate $\operatorname{Pic}(S) \simeq$ $\operatorname{Num}(S)$ with the pull back $l$ of the numerical class of a line in $\mathbb{P}^{2}$ and the classes $l_{i}$ of the 9 exceptional divisors. In this notation the class of any irreducible curve $\gamma$ on $S$ is either one of the $l_{i}$ or a class of the following type: $a l-a_{0} l_{0}-\ldots-a_{8} l_{8}$, for suitable integers $a \geq 1$ and $a_{i} \geq 0$, as $\gamma$ comes from an irreducible plane curve. The curve $\mathcal{C}$ in $\mathbb{P}^{2}$ gives rise to a curve $\overline{\mathcal{C}}$ on $S$ such that $\overline{\mathcal{C}} \equiv 3 l-l_{0}-\ldots-l_{8}$, moreover for any irreducible curve $\gamma$ on $S$, different from $l_{i}$, we have: $0 \leq \overline{\mathcal{C}} \gamma=3 a-a_{0}-\ldots-a_{8}$; it follows that the class $a l-a_{0} l_{0}-\ldots-a_{8} l_{8}$ of any irreducible curve $\gamma$ on $S$, different from $l_{i}$, must satisfy the condition: $3 a \geq a_{0}+\ldots+a_{8}$.

Very ampleness of $\mathcal{L}$ is equivalent to very ampleness of $\left|4 l-l_{0}-l_{1} \ldots-l_{8}\right|$ on $S$ and this will be established via Reider's method (see [D-L] Theorem 2.1). Let $M$ be a divisor on $S$ such that $4 l-l_{0}-l_{1} \ldots .-l_{8} \equiv K_{S}+M$. It is $M \equiv 7 l-2 l_{0}-2 l_{1} \ldots-2 l_{8}$. To be able to apply Reider's Theorem, $M$ must be big and nef with $M^{2} \geq 10$. Obviously $M^{2}=13$, moreover $M l_{i}=2$, for any $i$, and $M \gamma \geq a \geq 1$ for any other irreducible curve $\gamma$ on $S$, thanks to the above condition. It follows that $M$ is ample (Nakai-Moishezon criterion, see $[\mathrm{H}]$ pag. 365) and therefore big and nef.

Now, if $E$ is a candidate effective divisor that, according to Reider, could cause $K_{S}+M$ not to be very ample, it must be as in one of these cases:

1) $E \equiv l-\sum_{i=0}^{8} a_{i} l_{i}, E$ irreducible, $M E=1, \sum_{i=0}^{8} a_{i}=3,0 \leq a_{i} \leq 1$ for any $i$;

2) $E \equiv 2 l-\sum_{i=0}^{8} a_{i} l_{i}, E$ irreducible, $M E=2, \sum_{i=0}^{8} a_{i}=6$;

3) $E=E_{1}+E_{2}, E_{1} \neq E_{2}$, where each $E_{j}$ is irreducible, $E_{j} \equiv l-\sum_{i=0}^{8} a_{i j} l_{i}$, $M E=2, \sum_{i=0}^{8} a_{i j}=3,0 \leq a_{i j} \leq 1$ for any $i$ and $j$.

In all cases it is $E^{2} \leq-2$, not satisfying Reider's conditons, hence $\mathcal{L}$ is very ample.

Lemma 6.8. Let $\mathcal{E}_{y}$ be any vector bundle defined as above by $(*)$ with $y=3$ and $h=4$ (hence $w=7$ ). Let $\bar{f}$ be any fixed fibre of $Y$ and let $S_{1}:=\mathbb{P}\left(\mathcal{E}_{3 \mid \bar{f}}\right) \subset X$. Then:

$i)$ the generic element $S$ of the linear system $\left|T-S_{1}\right|$ is smooth and irreducible;

ii) any linear subsystem of $\left|T-S_{1}\right|$ consisting of elements which are all singular and or reducible has codimension at least two;

iii) the restriction map $|T| \rightarrow|T|_{\mid S}$ is surjective for any smooth $S \in\left|T-S_{1}\right|$;

iv) $T_{\mid S}$ is very ample for any generic smooth $S \in\left|T-S_{1}\right|$.

Proof. For simplicity let us write $\mathcal{E}$ instead of $\mathcal{E}_{3}$. By $(*)$ it follows that $h^{0}(Y, \mathcal{E})=8$. By tensorizing $(*)$ with $\mathcal{O}_{Y}(-\bar{f})$ we get that $h^{0}\left(Y, \mathcal{E} \otimes \mathcal{O}_{Y}(-\bar{f})\right)=h^{0}\left(X, T-S_{1}\right)=$ 3.

i) and $i i$ ). Let $\Lambda$ be any linear subsystem of $\left|T-S_{1}\right|$ such that every element of $\Lambda$ is singular or reducible. To prove $i$ ) and $i i$ ) we have to show that $\operatorname{dim}(\Lambda) \leq 0$. 
Recalling the proof of Proposition 3.4, we know that any element of $\left|T-S_{1}\right|$ is singular if and only if it is reducible and that $\Lambda \neq \emptyset$ if and only if there exists an effective divisor $D=a C_{0}+\rho^{*} B \in \operatorname{Pic}(Y)$, with $\operatorname{deg}(B)=b, a \geq 0, b \geq 0$, such that $T-S_{1}-\pi^{*} D$ is effective and, in this case, $\Lambda=\left|T-S_{1}-\pi^{*} D\right|+\left|\pi^{*} D\right|$ with $h^{0}\left(X, T-S_{1}-\pi^{*} D\right)=1$ and $h^{0}\left(X, \pi^{*} D\right)-1=\operatorname{dim}(\Lambda)$ or $h^{0}\left(X, \pi^{*} D\right)=1$ and $h^{0}\left(X, T-S_{1}-\pi^{*} D\right)-1=\operatorname{dim}(\Lambda)$.

From the exact sequence:

$0 \rightarrow C_{0}+f-\bar{f}-D \rightarrow \mathcal{E} \otimes \mathcal{O}_{Y}(-\bar{f}-D) \rightarrow\left(2 C_{0}+4 f-\bar{f}-D\right) \otimes \mathcal{I}_{W} \rightarrow 0$

we see that $h^{0}\left(X, T-S_{1}-\pi^{*} D\right)=h^{0}\left(Y, \mathcal{E} \otimes \mathcal{O}_{Y}(-\bar{f}-D)\right.$ can be positive only if $a \leq 1$.

Let us assume $a=1$. In this case we have to consider the exact sequence:

$0 \rightarrow-b f \rightarrow \mathcal{E} \otimes \mathcal{O}_{Y}(-\bar{f}-D) \rightarrow\left(C_{0}+3 f-b f\right) \otimes \mathcal{I}_{W} \rightarrow 0$.

As $P_{0} \cup s(W)$ are in general position it is easy to see that $h^{0}\left(Y,\left(C_{0}+3 f-b f\right) \otimes\right.$ $\left.\mathcal{I}_{W}\right)=0$ for any $b \geq 0$. If $b>0 h^{0}\left(X, T-S_{1}-\pi^{*} D\right)=h^{0}\left(Y, \mathcal{E} \otimes \mathcal{O}_{Y}(-\bar{f}-D)=0\right.$; if $b=0 h^{0}\left(X, T-S_{1}-\pi^{*} D\right)=h^{0}\left(Y, \mathcal{E} \otimes \mathcal{O}_{Y}(-\bar{f}-D)=1\right.$ and $h^{0}\left(X, \pi^{*} D\right)=$ $h^{0}(Y, D)=1$, so that $\operatorname{dim}(\Lambda)=0$ and we are done.

Let us assume $a=0$ (hence $b \geq 1$ ). In this case we have to consider the exact sequence:

$0 \rightarrow C_{0}-b f \rightarrow \mathcal{E} \otimes \mathcal{O}_{Y}(-\bar{f}-D) \rightarrow\left(2 C_{0}+3 f-b f\right) \otimes \mathcal{I}_{W} \rightarrow 0$.

As $P_{0} \cup s(W)$ are in general position it is easy to see that $h^{0}\left(Y,\left(2 C_{0}+3 f-\right.\right.$ $\left.b f) \otimes \mathcal{I}_{W}\right)=0$, moreover $h^{0}\left(Y, C_{0}-b f\right)=0$, so that $h^{0}\left(X, T-S_{1}-\pi^{*} D\right)=$ $h^{0}\left(Y, \mathcal{E} \otimes \mathcal{O}_{Y}(-\bar{f}-D)=0\right.$ and $\Lambda=\emptyset$.

iii) Let $S$ be a smooth element of $\left|T-S_{1}\right|$ and let us consider the exact sequence: $0 \rightarrow T-S \rightarrow T \rightarrow T_{\mid S} \rightarrow 0$. As $T-S=\pi^{*} \bar{f}$ we have that $h^{0}\left(X, \pi^{*} \bar{f}\right)=h^{0}(Y, \bar{f})=$ 2 and $h^{1}\left(X, \pi^{*} \bar{f}\right)=h^{1}(Y, \bar{f})=0$, so that $h^{0}(X, T-S)=2, h^{0}\left(S, T_{\mid S}\right)=8-2=6$ and the map $H^{0}(X, T) \rightarrow H^{0}\left(S, T_{\mid S}\right)$ is surjective.

iv) $S$, being a smooth generic element of $\left|T-\pi^{*} \bar{f}\right|$, is isomorphic to the blow up of $Y$ at $\operatorname{deg}\left\{c_{2}\left[\mathcal{E} \otimes \mathcal{O}_{Y}(-\bar{f})\right]\right\}=8$ distinct points (see the proof of Lemma 6.4$, iii $)$ ). Let $C_{0}^{\prime}, f^{\prime}, l_{1}, \ldots ., l_{8}$ be the generators of $\operatorname{Num}(S)$ as in Lemma 6.4 iii) for $S_{2}$. The Wu-Chern relation for $\mathcal{E} \otimes \mathcal{O}_{Y}(-\bar{f})$ implies that $\left(T-\pi^{*} \bar{f}\right)^{2}=$ $\pi^{*}\left\{c_{1}\left[\mathcal{E} \otimes \mathcal{O}_{Y}(-\bar{f})\right]\right\}\left(T-\pi^{*} \bar{f}\right)-c_{2}\left[\mathcal{E} \otimes \mathcal{O}_{Y}(-\bar{f})\right]$. Hence $\left(T-\pi^{*} \bar{f}\right)_{\mid S} \equiv\left(\pi_{\mid S}\right)^{*}\left\{c_{1}[\mathcal{E} \otimes\right.$ $\left.\left.\mathcal{O}_{Y}(-\bar{f})\right]\right\}-l_{1} \ldots-l_{8} \equiv\left(\pi_{\mid S}\right)^{*}\left(3 C_{0}+5 f-2 f\right)-l_{1} \ldots-l_{8}$ and $T_{\mid S} \equiv\left(\pi_{\mid S}\right)^{*}\left(3 C_{0}+\right.$ $5 f-f)-l_{1} \ldots-l_{8} \equiv 3 C_{0}^{\prime}+4 f^{\prime}-l_{1} \ldots-l_{8}$.

As $Y$ is the blow up of $\mathbb{P}^{2}$ at one point $P_{0}, S$ is isomorphic to the blow up of $\mathbb{P}^{2}$ at 9 distinct points, so we can also generate $\operatorname{Num}(S)$ with the pull back $l$ of the numerical class of a line in $\mathbb{P}^{2}$ and the classes of the 9 exceptional divisors. If $l_{0}$ is the class of the pull back of the exceptional divisor of the blow up of $\mathbb{P}^{2}$ at $P_{0}$ we have $C_{0}^{\prime} \equiv l_{0}$ and $f^{\prime} \equiv l-l_{0}$ so that: $T_{\mid S} \equiv 4 l-l_{0}-l_{1} \ldots-l_{8}$.

To show that $T_{\mid S}$ is very ample we can apply Lemma $6.7: h^{0}\left(S, T_{\mid S}\right)=6$ by iii); $\left|T_{\mid S}\right|$ does not have base points, because $|T|$ does not have base points by Lemma $6.4 \mathrm{ii})$ and $|T| \rightarrow\left|T_{\mid S}\right|$ is surjective; the 9 distinct points lie on a smooth cubic because the zero-locus of a generic section of $\mathcal{E} \otimes \mathcal{O}_{Y}(-\bar{f})$ is a set of 8 distinct points belonging to a generic element of the linear system $\left|\left(2 C_{0}+3 f\right) \otimes \mathcal{I}_{W}\right|=\left|\left(3 l-l_{0}\right) \otimes \mathcal{I}_{W}\right|$ on $Y$, (see the proof of Lemma 6.4, iii)) corresponding to a smooth plane cubic curve passing through $P_{0} \cup s(W)$, where $s: Y \rightarrow \mathbb{P}^{2}$ is the blow up.

Theorem 6.9. Let $\mathcal{E}_{y}$ be any vector bundle defined as above by $(*)$ with $y=3$ and $h=4$ (hence $w=7$ ), then $\mathcal{E}_{3}$ is very ample. 
Proof. As before, let us write $\mathcal{E}$ instead of $\mathcal{E}_{3}$. Let $T$ be the tautological divisor of $X=\mathbb{P}(\mathcal{E})$ and let $\xi$ be any fixed element of $X^{[2]}$. We have to prove that $|T|$ separates $\xi$. By Lemmas 6.5 and 6.4 we know that we have to consider only the cases when $\xi$ is a couple of distinct points $P, Q$ projecting on different fibres of $Y$.

We want to apply Proposition 3.5, so that we will prove that assumptions $\alpha$ ) and $\gamma$ ) of Proposition 3.5 are satisfied with $A \equiv C_{0}+x f, x>>0$, and $B \equiv f$. It is easy to see that $\gamma$ ) is true for $x>>0$, so we have to prove that $\alpha$ ) is true for any couple of distinct points $P, Q \in X$. In other words, we have to show that there exists a section $\tau \in|T|$ such that $(\tau)_{0}$ passes through $P$ and does not pass through $Q$. Of course we can change the role of $P$ and $Q$ to separate $Q$ from $P$.

Let us consider $\pi(Q)$ and the fibre $f_{Q}$ of $Y$ passing through $\pi(Q)$. Let us choose a smooth surface $S_{2} \in\left|T+\pi^{*} A\right|$ passing through $P$ and not through $Q$ (recall that $T+\pi^{*} A$ is very ample for $x>>0$ ) and a smooth $S_{1}:=\pi^{-1}\left(f_{Q}\right)$; so $\alpha$ ) $i$ ) is fullfilled, moreover we can assume that $S_{2}$ cuts $S_{1}$ transversely along a smooth rational curve $C$ as in the proof of Lemma 6.5.

As $T_{\mid S_{1}}$ is very ample by Lemma $6.4 \mathrm{i}$ ), we can choose a section $\sigma_{1} \in\left|T_{\mid S_{1}}\right|$ such that $\left(\sigma_{1}\right)_{0}$ is smooth, does not pass through $Q$ and cuts $C$ at 4 distinct points $R_{1}, \ldots, R_{4}$ (see the proof of Lemma 6.5 ) so that $\alpha$ ) $i i$ ) is fulfilled.

To get $\alpha) i i i)$ and $i v)$ we look for a section $\sigma_{2} \in H^{0}\left(S_{2}, T_{\mid S_{2}}\right)$ whose zero locus passes through $P, R_{1}, \ldots, R_{4}$ and does not contain $C$. By Lemma 6.4 iii) we know that $S_{2}$ is isomorphic to the the blow up of $Y$ at $5 x+12$ distinct points, hence to the blow up of $\mathbb{P}^{2}$ at $5 x+13$ distinct points; if we generate $\operatorname{Num}\left(S_{2}\right)$ with the pull back $l$ of the generator of $\operatorname{Pic}\left(\mathbb{P}^{2}\right)$, the pull back $l_{0}$ of $C_{0} \in Y$ and the classes of the exceptional divisors, we have that $T_{\mid S_{2}} \equiv(x+5) l-(x+1) l_{0}-l_{1} \cdots-l_{5 x+12}$, $|T| \simeq\left|T_{\mid S_{2}}\right|$ and $h^{0}\left(S_{2}, T_{\mid S_{2}}\right)=h^{0}(X, T)=h^{0}(Y, \mathcal{E})=8$.

Let us consider the linear subspace $H^{0}\left(S_{2}, T_{\mid S_{2}} \otimes \mathcal{I}_{P}\right)$ of $H^{0}\left(S_{2}, T_{\mid S_{2}}\right)$ given by sections whose zero locus contains $P ; \operatorname{dim}\left[H^{0}\left(S_{2}, T_{\mid S_{2}} \otimes \mathcal{I}_{P}\right)\right]=7$, (recall that $|T| \simeq$ $|T|_{\mid S_{2}}=\left|T_{\mid S_{2}}\right|$ has no base points by Lemma $\left.\left.6.4 \mathrm{ii}\right)\right)$. Let us consider the restriction $\rho_{P}$ of the natural map $\rho: H^{0}\left(S_{2}, T_{\mid S_{2}}\right) \rightarrow H^{0}\left(C, T_{\mid C}\right) \simeq H^{0}\left(\mathbb{P}^{1}, \mathcal{O}_{\mathbb{P}^{1}}(4)\right) \simeq \mathbb{C}^{5}$ to $H^{0}\left(S_{2}, T_{\mid S_{2}} \otimes \mathcal{I}_{P}\right)$.

We claim that $\rho$ is surjective. Indeed, consider first the structure sequence of $S_{2}$ on $X$, tensored with $T-S_{1}$ :

$0 \rightarrow T-S_{1}-S_{2} \rightarrow T-S_{1} \rightarrow\left(T-S_{1}\right)_{\mid S_{2}} \rightarrow 0$. It is: $h^{1}\left(X, T-S_{1}\right)=0$ by Proposition $6.2 \mathrm{iii})$ and $h^{2}\left(X, T-S_{1}-S_{2}\right)=h^{2}\left(X,-\pi^{*}\left(A+f_{Q}\right)\right)=h^{2}(Y,-A-$ $\left.f_{Q}\right)=h^{0}\left(Y, K_{Y}+A+f_{Q}\right)=0$, hence $h^{1}\left(S_{2},\left(T-S_{1}\right)_{\mid S_{2}}\right)=0$.

Let us then consider the structure sequence of $C$ on $S_{2}$ tensored with $T_{\mid S_{2}}$. As $h^{1}\left(S_{2},\left(T-S_{1}\right)_{\mid S_{2}}\right)=h^{1}\left(S_{2}, T_{\mid S_{2}}-C\right)=0$ it follows that $\rho$ is surjective, so that our claim is proved, moreover $\operatorname{ker}(\rho)=H^{0}\left(S_{2}, T_{\mid S_{2}}-C\right)$. As $3=h^{0}\left(X, T-S_{1}\right)=$ $h^{0}\left(S_{2},\left(T-S_{1}\right)_{\mid S_{2}}\right)=h^{0}\left(S_{2}, T_{\mid S_{2}}-C\right)$, it follows that $\operatorname{dim}[\operatorname{ker}(\rho)]=3$.

Now let us consider the following two cases.

Case 1: let us assume that $P$ is in the base locus of $\left|T_{\mid S_{2}}-C\right|=\left|\left(T-S_{1}\right)_{\mid S_{2}}\right|=$ $\left|T-S_{1}\right|_{\mid S_{2}}$, hence in the base locus of $\left|T-S_{1}\right|$ because $|T| \simeq|T|_{\mid S_{2}}=\left|T_{\mid S_{2}}\right|$. If $Q$ is not in the base locus of $\left|T-S_{1}\right|$, then there exists an element $\widetilde{S} \in\left|T-S_{1}\right|=\left|T-\pi^{*} f\right|$ passing through $P$ and not passing through $Q$. Let us pick $S_{1}^{\prime}:=\pi^{-1}(\bar{f})$, where $\bar{f}$ is a fibre of $Y$ different from $f_{Q}$ and we get an element $\widetilde{S} \cup S_{1}^{\prime} \in\left|T-\pi^{*} f\right|+\left|\pi^{*} f\right| \subseteq|T|$ separating $P$ from $Q$ without using Proposition 3.5. If $Q$ is in the base locus of $\left|T-S_{1}\right|$, let us pick a generic smooth surface $S \in\left|T-S_{1}\right|$, obviously passing through 
$P$ and $Q$, and existing by Lemma $6.8 i$ ) and $i i$ ). Now, by Lemma $6.8 \mathrm{iii}$ ) and $i v$ ) we can separate $P$ from $Q$ by $|T|$ directly, without using Proposition 3.5.

Case 2: let us assume that $P$ is not in the base locus of the linear system $\left|T_{\mid S_{2}}-C\right|=\left|\left(T-S_{1}\right)_{\mid S_{2}}\right|=\left|T-S_{1}\right|_{\mid S_{2}}$. Then there exists at least an element of $\left|T_{\mid S_{2}}-C\right|$ whose zero-locus does not pass through $P$, hence $H^{0}\left(S_{2}, T_{\mid S_{2}}\right)_{P} \nsupseteq$ $H^{0}\left(S_{2}, T_{\mid S_{2}}-C\right)=\operatorname{ker}(\rho)$ and $\operatorname{dim}\left[\operatorname{ker}\left(\rho_{P}\right)\right]=\operatorname{dim}\left[H^{0}\left(S_{2}, T_{\mid S_{2}}\right)_{P} \cap H^{0}\left(S_{2}, T_{\mid S_{2}}-\right.\right.$ $C)]=2$, (a priori $\operatorname{dim}\left[\operatorname{ker}\left(\rho_{P}\right)\right]=\{2,3\}$ ). Therefore $\rho_{P}$ is surjective too and we can choose a section $\sigma_{2} \in\left|T_{\mid S_{2}}\right|$ whose zero locus passes through $P, R_{1}, \ldots, R_{4}$, not containing $C$. In this case we can conclude as in the proof of Lemma 6.5: as $H^{0}(X, T)=H^{0}\left(S_{2}, T_{\mid S_{2}}\right)$ we get that there exists $\tau \in|T|$ such that $\tau_{\mid S_{2}}=\sigma_{2}$ and $(\tau)_{0}$ does not contain $S_{1}$ because $\left(\sigma_{2}\right)_{0}$ does not contain $C$. Hence $\sigma_{1}^{\prime}:=\tau_{\mid S_{1}}$ is a non zero section of $\left|T_{\mid S_{1}}\right|$ and we have $\sigma_{1}^{\prime} \in<\sigma_{1}>$ because $\sigma_{1}^{\prime}$ and $\sigma_{1}$ cut the same divisor $R_{1}+R_{2}+R_{3}+R_{4}$ on $C$ and $\left|T_{\left|S_{1}\right|}\right|_{\mid C} \simeq\left|T_{\left|S_{1}\right|}\right|$ by Lemma $6.4 i$ ). By choosing suitably the generator of $\left\langle\sigma_{1}\right\rangle$ we can assume that $\tau_{\mid S_{1}}=\sigma_{1}$ so that $\alpha) i i i)$ and $\alpha) i v)$ are fulfilled a fortiori by choosing $\sigma:=\tau_{\mid S ! \cup S_{2}}$.

\section{Existence AND NON EXIstence Of SOME 3-FOldS}

The study of linearly normal projective manifolds of low degree got a boost as a result of classical adjunction theory, as developed by Sommese and his collaborators. The approach consists of three phases: enumeration of all possible manifolds of given degree according to their adjunction theoretic structure and values of numerical characters; investigation of actual effective existence of elements appearing in the compiled lists; study of the Hilbert scheme of existing manifolds (see [B-B-2] for details). In [F-L-1], [F-L-2], [B-B-1], such a study is conducted for degree, respectively, $9,10,11$. In all three papers the existence of members of a particular family of 3 -fold scrolls was left as an open problem. They are scrolls of the form $X:=\mathbb{P}\left(\mathcal{E}_{y}\right)$, of degree $\left[c_{1}\left(\mathcal{E}_{y}\right)\right]^{2}-c_{2}\left(\mathcal{E}_{y}\right)=13-y$, where $\mathcal{E}_{y}$ is a rank 2 vector bundle over $Y=\mathbf{F}_{1}$, having $c_{1}\left(\mathcal{E}_{y}\right) \equiv 3 C_{0}+5 f$ and $c_{2}\left(\mathcal{E}_{y}\right)=8+y$, with $y=2,3,4$.

The analysis conducted in Section 6 gives immediately the following::

Corollary 7.1. There exist linearly normal 3 -folds $\mathbb{P}\left(\mathcal{E}_{y}\right), y=3,2$, where $\mathcal{E}_{y}$ is a rank two vector bundle given by $(*)$, embedded as linear scrolls over $\mathbf{F}_{1}$, with $c_{1}\left(\mathcal{E}_{y}\right) \equiv 3 C_{0}+5 f, c_{2}\left(\mathcal{E}_{y}\right)=8+y$ and degree, respectively, 10 and 11 .

Proof. Apply Theorem 6.6 and Theorem 6.9, by recalling that vector bundles $\mathcal{E}_{y}$ defined by $(*)$ have the prescribed Chern classes.

In fact Theorem. 6.6 proves the existence of other 3-folds of the same type. It is natural to ask if rank 2 very ample vector bundles over $Y$ can be defined by using $(*)$ for other values of $y$. The answer is negative for $y=4, h=4$. In this case it is possible to prove that there is a smooth surface $S \in\left|T-\pi^{*} D\right|$, with $D \equiv C_{0}+f$, such that $S$ is isomorphic to the blow up of $\mathbb{P}^{2}$ at 9 distinct points in general position and $T_{\mid S} \simeq 4 l-2 l_{0}-l_{1} \ldots-l_{8}$ (with the usual notation). If we consider an existing smooth plane cubic curve passing through $P_{0}, \ldots, P_{8}$ we have that this curve gives rise to a smooth elliptic curve $C$ on $S$ such that $T_{\mid C}$ is not very ample $\left(\operatorname{deg}\left(T_{\mid S} C\right)=\operatorname{deg}\left(T_{\mid C}\right)=2\right)$, so that $T$ can not be very ample. In the same way it is possible to show that the same approach is not successful for $y=4$, $h=3$ and also for $y=4$ and $h=5$.

On the other hand it is easy to prove that there exists a very ample rank 2 vector bundles $\mathcal{E}_{-3}$ over $Y$ having $c_{1}\left(\mathcal{E}_{-3}\right) \equiv 3 C_{0}+5 f$ and $c_{2}\left(\mathcal{E}_{-3}\right)=5$ : indeed 
one can simply take $\mathcal{E}_{-3}$ to be the direct sum of the two very ample line bundles $L \equiv C_{0}+2 f$ and $M \equiv 2 C_{0}+3 f$.

The following necessary condition for the very ampleness of rank 2 vector bundles $\mathcal{E}$, over any $\mathbf{F}_{e}$, having $c_{1}(\mathcal{E}) \equiv 3 C_{0}+t f$ for some integer $t$, can be established.

Proposition 7.2. Let $\mathcal{E}$ be a very ample rank 2 vector bundle over $\mathbf{F}_{e}$, such that $c_{1}(\mathcal{E}) \equiv 3 C_{0}+t f$ and $c_{2}(\mathcal{E})=k$. Then: $h^{0}\left(\mathbf{F}_{e}, \mathcal{E}\right) \geq 7, t \geq 3 e+1, k+e>t$ and there exists an exact sequence $0 \rightarrow L \rightarrow \mathcal{E} \rightarrow M \rightarrow 0$ where $L$ and $M$ are line bundles such that $L \equiv 2 C_{0}+(2 t-2 e-k) f$, and $M \equiv C_{0}+(k-t+2 e) f$.

Proof. As $\mathcal{E}$ is very ample, $\left(\mathbb{P}(\mathcal{E}), \mathcal{O}_{\mathbb{P}(\mathcal{E})}(1)\right)$ is a scroll over $\mathbf{F}_{e}$ and it is known that there are no such scrolls in $\mathbb{P}^{r}$ when $r \leq 5$, see $[\mathrm{O}]$. Moreover, if $\mathcal{E}$ is very ample then $c_{1}(\mathcal{E})$ is ample, so that $t \geq 3 e+1$, see $\left[\mathrm{H}\right.$, Corollary 2.18]. Let $\rho: \mathbf{F}_{e} \rightarrow \mathbb{P}^{1}$ be the usual natural projection. The restriction $\mathcal{E}_{\left.\right|_{f}}$ to any fibre $f$ of $\rho$ must also be very ample. But $\mathcal{E}_{\mid f}=\mathcal{O}_{\mathbb{P}^{1}}(a) \oplus \mathcal{O}_{\mathbb{P}^{1}}(b)$ with $a+b=3$ as $c_{1}(\mathcal{E}) f=3$, therefore the only possibility is $a=2, b=1$ for any fibre. By [B, p. 155], Theorem 1, there exists an exact sequence $0 \rightarrow L \rightarrow \mathcal{E} \rightarrow M \rightarrow 0$ such that $L+M \equiv c_{1}(\mathcal{E}), L M=c_{2}(\mathcal{E})=k$ and $L=\rho^{*}\left[\rho_{*}\left(\mathcal{E} \otimes \mathcal{O}_{\mathbf{F}_{e}}\left(-2 C_{0}\right)\right] \otimes \mathcal{O}_{\mathbf{F}_{e}}\left(2 C_{0}\right)\right.$. In fact the zero-dimensional scheme $Z$ which is involved in the exact sequence of $[\mathrm{B}]$ in this case is empty. Indeed $\rho(\operatorname{Supp}(Z))$ would coincide with the projection of the jumping lines for $\mathcal{E}$, but in this case $\mathcal{E}$ is uniform on the ruling. It follows that $L \equiv 2 C_{0}+\lambda f$ for some integer $\lambda$, and $M \equiv C_{0}+(t-\lambda) f$. As $L M=c_{2}(\mathcal{E})=k$, it is $\lambda=2 t-2 e-k$. The very ampleness of $\mathcal{E}$ implies that $M$ is ample, hence $t-(2 t-2 e-k)>e$, i.e. $k+e>t$.

Proposition 7.2 shows that, if a vector bundle over $Y$ as $\mathcal{E}_{4}$ is very ample, then it is the extension of two line bundles. However this fact does not help to prove the very ampleness of a rank 2 vector bundle by our techniques.

We can also establish a non-existence result that settles one more existence question left open in [B-B-1].

Corollary 7.3. There does not exist any linearly normal 3 -fold $X=\mathbb{P}(\mathcal{E})$, embedded in $\mathbb{P}^{7}$ as linear scroll over $Y=\mathbf{F}_{1}$, with degree 11 and sectional genus 5 .

Proof. By contradiction, let us assume that $X$ exists in $\mathbb{P}^{7}$, hence $h^{0}(X, T)=8$ because $X$ is supposed to be linearly normal. As in the proof of Lemma 6.4 iii), let us consider a divisor $A \equiv C_{0}+x f$ on $Y$ with $x>>0$ and a smooth surface $S_{2}$ such that $S_{2}$ is isomorphic to the blow up of $\mathbb{P}^{2}$ at $5 x+12$ distinct points and $H^{0}(X, T)=H^{0}\left(S_{2}, T_{\mid S_{2}}\right)$. Recall that we are assuming that $\mathcal{E}$ is very ample so that $\mathcal{E} \otimes A$ is very ample too; note that here the position of the points on $\mathbb{P}^{2}$ it is not important. As $T_{\mid S_{2}} \equiv(x+5) l-(x+1) l_{0}-l_{1} \cdots-l_{5 x+11}$ we have that $h^{0}\left(S_{2}, T_{\mid S_{2}}\right) \geq 9$ : contradiction!

\section{REFERENCES}

[A-B-B] A.Alzati-M.Bertolini-G.M.Besana: "Numerical criteria for very ampleness of divisors on projective bundles over an elliptic curve" Can. J. Math. 48(6) (1996) p.1121-1137.

[B] J.E.Brosius: "Rank-2 Vector Bundles on a Ruled Surface I" Math. Ann. 265 (1983) p.155-168.

[B-B-1] G.Besana-A.Biancofiore: "Degree 11 Manifolds of Dimension Greater or equal to 3" Forum Math. 17 (5) (2005) p.711-733.

[B-B-2] G.Besana-A.Biancofiore: "Numerical Constraints for Embedded Projective Manifolds" Forum Math. 17 (4) (2005) p.613-636. 
[B-F] G.M.Besana-M.L.Fania: "The dimension of the Hilbert scheme of special threefolds". Comm. Algebra 33 (10) (2005), p.3811-3829.

[B-S] M.Beltrametti-A.J.Sommese: "The adjunction theory of complex projective varieties" Expositions in Mathematics, vol 16, De Gruyter, 1995.

[B-D-S] M.Beltrametti-S.Di Rocco-A.J.Sommese: "On generation of jets for vector bundles" Rev. Mat. Complut. 12 (1) (1999) p.27-45.

[Bu] D.C.Butler: "Normal generation of vector bundles over a curve" J. Differential Geom. 39(1) (1994) p.1-34.

[C-M] C. Ciliberto-R.Miranda: "Degeneration of planar linear systems" J. reine angew. Math. 501 (1998) p.191-220.

[D-L] G.F. del Busto-R.Lazarsfeld: "Complex algebraic geometry (Park City, UT, 1993)", IAS/Park City Math. Ser., 3, p.161-219, Amer. Math. Soc., Providence, RI, 1997.

[F-L-1] L.Fania-E.L.Livorni: "Degree Nine Manifolds of Dimension Greater than or Equal to 3" Math. Nachr. 169 (1994) p.117-134.

[F-L-2] L.Fania-E.L.Livorni: "Degree ten manifolds of dimension $n$ greater than or equal to 3" Math. Nachr. 188 (1997) p.79-108.

[G-H] P.Griffiths-J.Harris: "Principles of Algebraic Geometry" Wiley Interscience New York 1994.

[H] R.Hartshorne: "Algebraic Geometry" Springer Verlag 1977.

[H-R] A.Holme-J.Roberts: "On the embeddings of projective varieties". Algebraic geometry (Sundance, UT, 1986), Lecture Notes in Math. 1311, Springer, Berlin, (1988) p.118-146.

[M] Y.Miyaoka: "The Chern class and Kodaira dimension of a minimal variety" In: Algebraic Geometry, Sendai 1985, Adv. Stud. Pure Math. 10, Amer. Math. Soc., (1987), p.449-476.

[O] G.Ottaviani: "On 3-Folds in $\mathbb{P}^{5}$ which are Scrolls" Ann. Sc. Norm. Sup. Pisa, Serie IV, 19 (3) (1992) p.451-471.

Dipartimento di Matematica Univ. di Milano, via C. Saldini 50 20133-Milano (Italy)

E-mail address: alberto.alzati@unimi.it

College of Computing and Digital Media, De Paul University - 243 S Wabash Chicago IL 60604 USA

E-mail address: gbesana@cs.depaul.edu 